\title{
Synthesis and structures of complexes demonstrating the coordinative versatility of the 2,4-diimino-3-phosphinopentane anion
}

\author{
Neil Burford $*^{a}$, Mark D'eon ${ }^{a}$, Paul J. Ragogna, ${ }^{a}$ Robert McDonald and Michael J. Ferguson ${ }^{b}$
}

${ }^{a}$ Department of Chemistry, Dalhousie University, Halifax, NS, B3H 4J3, CANADA

${ }^{b} \mathrm{X}$-ray Crystallography Laboratory, Department of Chemistry, University of Alberta, Edmonton Alberta, T6G 2G2, CANADA

\section{Supporting Information Package}

Figure S1: Solid state structure of $\mathbf{5} \mathbf{A l C l}_{2}$. Ellipsoids are $50 \%$ probability, hydrogen atoms are removed for clarity and the full numbering scheme is highlighted.

Table S1: Crystal data and structure refinement for $\mathbf{5} \mathbf{A I C l}_{\mathbf{2}}$.

Table S2: Atomic coordinates ( $\left.\mathrm{x} 10^{4}\right)$ and equivalent isotropic displacement parameters $\left(\AA^{2} \mathrm{x} 10^{3}\right)$

for $\mathbf{5 A l}$. U(eq) is defined as one third of the trace of the orthogonalized $\mathrm{U}^{\mathrm{ij}}$ tensor.

Table S3: Bond lengths $[\AA]$ and angles $\left[{ }^{\circ}\right]$ for $\mathbf{5} \mathbf{A I C l}_{2}$.

Table S4: Anisotropic displacement parameters $\left(\AA^{2} \mathrm{x} 10^{3}\right)$ for $\mathbf{5} \mathbf{A l C l}_{2}$. The anisotropic

displacement factor exponent takes the form: $-2 \pi^{2}\left[h^{2} a^{* 2} U^{11}+\ldots+2 h k a^{*} b^{*} U^{12}\right]$

Table S5: Torsion angles $\left[{ }^{\circ}\right]$ for $\mathbf{5} \mathbf{A l C l}_{2}$.

Figure S2: Solid state structure of $\mathbf{5 L i}$. Ellipsoids are 50\% probability, hydrogen atoms and toluene solvate are removed for clarity. Numbering scheme of core atoms is included.

Table S6: Crystal data and structure refinement for $5 \mathbf{L i}$.

Table S7: Atomic coordinates ( x 10 $)$ and equivalent isotropic displacement parameters $\left(\AA^{2} \mathrm{x} 10^{3}\right)$

for $5 \mathbf{L i}$. $U(e q)$ is defined as one third of the trace of the orthogonalized $U^{i j}$ tensor.

Table S8: Bond lengths $[\AA]$ and angles $\left[^{\circ}\right]$ for $\mathbf{5 L i}$.

Table S9: Anisotropic displacement parameters $\left(\AA^{2} \mathrm{x} 10^{3}\right)$ for $\mathbf{5 L i}$. The anisotropic displacement factor exponent takes the form: $-2 \pi^{2}\left[\mathrm{~h}^{2} \mathrm{a}^{* 2} \mathrm{U}^{11}+\ldots+2 \mathrm{~h} \mathrm{k} \mathrm{a*} \mathrm{b}^{*} \mathrm{U}^{12}\right]$

Figure S3: Solid State Structure of $\mathbf{6 S b}$. Ellipsoids are 50\% probability, hydrogen atoms are removed for clarity. This highlights the complete numbering scheme and includes the $n$-pentane solvate.

Table S10: Crystal data and structure refinement for $\mathbf{6 S b}$.

Table S11: Atomic coordinates ( x 104) and equivalent isotropic displacement parameters $\left(\AA^{2} \times 10^{3}\right)$

for $\mathbf{6 S b}$. $U(\mathrm{eq})$ is defined as one third of the trace of the orthogonalized $\mathrm{U}^{\mathrm{ij}}$ tensor.

Table S12: Bond lengths $[\AA]$ and angles $\left[{ }^{\circ}\right]$ for $\mathbf{6 S b}$.

Table S13: Anisotropic displacement parameters $\left(\AA^{2} \times 10^{3}\right)$ for $\mathbf{6 S b}$. The anisotropic displacement factor exponent takes the form: $-2 \pi^{2}\left[h^{2} a^{* 2} U^{11}+\ldots+2 h k a^{*} b^{*} U^{12}\right]$ 


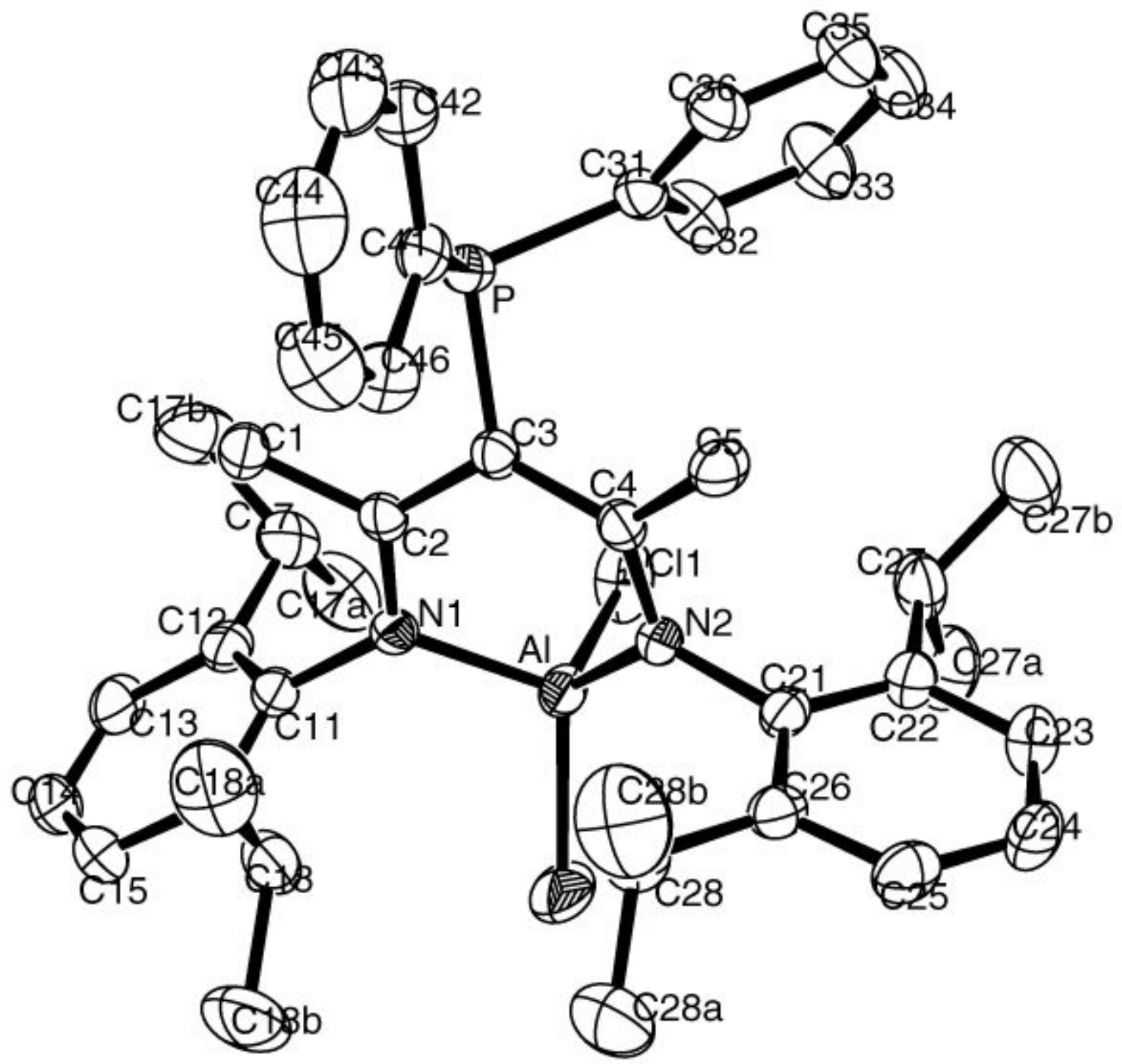

Figure S1: Solid state structure of $\mathbf{5} \mathbf{A l C l}_{\mathbf{2}}$. Ellipsoids are $50 \%$ probability, hydrogen atoms are removed for clarity and the full numbering scheme is highlighted. 
Table S1. Crystal data and structure refinement for $\mathbf{5} \mathbf{A l C l}_{\mathbf{2}}$.

Identification code

Empirical formula

Formula weight

Temperature

Wavelength

Crystal system

Space group

Unit cell dimensions

Volume

$\mathrm{Z}$

Density (calculated)

Absorption coefficient

$\mathrm{F}(000)$

Crystal size

Theta range for data collection

Index ranges

Reflections collected

Independent reflections

Completeness to theta $=26.49^{\circ}$

Absorption correction

Max. and min. transmission

Refinement method

Data / restraints / parameters

Goodness-of-fit on $\mathrm{F}^{2}$

Final $\mathrm{R}$ indices [I $>2 \operatorname{sigma}(\mathrm{I})]$

$\mathrm{R}$ indices (all data)

Largest diff. peak and hole dal0386

C41 H50 Al Cl2 N2 P

699.68

193(2) K

$0.71073 \AA$

Orthorhombic

Pbca

$\mathrm{a}=18.3867(11) \AA \quad \alpha=90^{\circ}$.

$\mathrm{b}=20.2465(12) \AA \quad \beta=90^{\circ}$.

$\mathrm{c}=21.2320(13) \AA \quad \gamma=90^{\circ}$.

7904.0(8) $\AA^{3}$

8

$1.176 \mathrm{Mg} / \mathrm{m}^{3}$

$0.257 \mathrm{~mm}^{-1}$

2976

$0.32 \times 0.16 \times 0.13 \mathrm{~mm}^{3}$

1.78 to $26.49^{\circ}$.

$-22<=\mathrm{h}<=23,-25<=\mathrm{k}<=25,-26<=\mathrm{l}<=26$

48531

$8131[\mathrm{R}($ int $)=0.1194]$

$99.4 \%$

Empirical

0.9674 and 0.9223

Full-matrix least-squares on $\mathrm{F}^{2}$

$8131 / 0 / 424$

1.007

$\mathrm{R} 1=0.0564, \mathrm{wR} 2=0.1175$

$\mathrm{R} 1=0.1092, \mathrm{wR} 2=0.1380$

0.476 and -0.474 e. $\AA^{-3}$ 
Table S2. Atomic coordinates ( $\mathrm{x} 10^{4}$ ) and equivalent isotropic displacement parameters $\left(\AA^{2} \times 10^{3}\right)$ for $\mathbf{5} \mathbf{A l C l}_{2}$. U(eq) is defined as one third of the trace of the orthogonalized $\mathrm{U}^{\mathrm{ij}}$ tensor.

\begin{tabular}{|c|c|c|c|c|}
\hline & $\mathrm{x}$ & $\mathrm{y}$ & $\mathrm{z}$ & $\mathrm{U}(\mathrm{eq})$ \\
\hline $\mathrm{Cl}(1)$ & $7423(1)$ & 1833(1) & $6205(1)$ & $53(1)$ \\
\hline $\mathrm{Cl}(2)$ & $7080(1)$ & 763(1) & $7468(1)$ & $61(1)$ \\
\hline $\mathrm{P}$ & 4781(1) & $2226(1)$ & 5401(1) & $25(1)$ \\
\hline $\mathrm{Al}$ & $6620(1)$ & 1352(1) & $6758(1)$ & $29(1)$ \\
\hline $\mathrm{N}(1)$ & $6027(1)$ & $858(1)$ & $6227(1)$ & $25(1)$ \\
\hline $\mathrm{N}(2)$ & $5927(1)$ & 1960(1) & $7035(1)$ & $24(1)$ \\
\hline $\mathrm{C}(1)$ & $5128(2)$ & $808(1)$ & $5377(1)$ & $33(1)$ \\
\hline $\mathrm{C}(2)$ & $5511(2)$ & $1172(1)$ & $5903(1)$ & $24(1)$ \\
\hline$C(3)$ & $5276(1)$ & $1825(1)$ & $6043(1)$ & $24(1)$ \\
\hline $\mathrm{C}(4)$ & $5403(2)$ & $2147(1)$ & $6635(1)$ & $25(1)$ \\
\hline$C(5)$ & 4921(2) & 2712(1) & $6827(1)$ & $33(1)$ \\
\hline $\mathrm{C}(11)$ & $6170(2)$ & $162(1)$ & 6092(1) & $29(1)$ \\
\hline $\mathrm{C}(12)$ & $6621(2)$ & $-17(1)$ & $5592(1)$ & $33(1)$ \\
\hline $\mathrm{C}(13)$ & $6730(2)$ & $-691(2)$ & $5479(2)$ & $42(1)$ \\
\hline $\mathrm{C}(14)$ & $6407(2)$ & $-1157(2)$ & $5852(2)$ & $48(1)$ \\
\hline $\mathrm{C}(15)$ & $5974(2)$ & $-975(2)$ & $6348(2)$ & $46(1)$ \\
\hline$C(16)$ & $5855(2)$ & $-315(1)$ & $6489(1)$ & $35(1)$ \\
\hline $\mathrm{C}(17)$ & $6995(2)$ & $481(2)$ & $5165(2)$ & $40(1)$ \\
\hline $\mathrm{C}(17 \mathrm{~A})$ & $7816(2)$ & $461(2)$ & $5264(2)$ & $64(1)$ \\
\hline $\mathrm{C}(17 \mathrm{~B})$ & $6814(3)$ & $373(2)$ & $4469(2)$ & $68(1)$ \\
\hline $\mathrm{C}(18)$ & $5378(2)$ & $-124(2)$ & $7046(2)$ & $47(1)$ \\
\hline $\mathrm{C}(18 \mathrm{~A})$ & 4591(2) & $-29(2)$ & $6856(2)$ & $71(1)$ \\
\hline $\mathrm{C}(18 \mathrm{~B})$ & $5436(3)$ & $-614(2)$ & $7587(2)$ & $82(2)$ \\
\hline $\mathrm{C}(21)$ & $5945(2)$ & $2288(1)$ & 7651(1) & $27(1)$ \\
\hline $\mathrm{C}(22)$ & $6373(2)$ & 2853(1) & $7735(1)$ & $34(1)$ \\
\hline $\mathrm{C}(23)$ & $6324(2)$ & $3181(2)$ & $8308(2)$ & $44(1)$ \\
\hline $\mathrm{C}(24)$ & $5870(2)$ & $2963(2)$ & $8780(2)$ & $44(1)$ \\
\hline $\mathrm{C}(25)$ & $5466(2)$ & $2403(2)$ & $8695(1)$ & $38(1)$ \\
\hline$C(26)$ & $5501(2)$ & $2046(1)$ & $8135(1)$ & $31(1)$ \\
\hline $\mathrm{C}(27)$ & $6898(2)$ & $3118(2)$ & $7239(2)$ & $42(1)$ \\
\hline $\mathrm{C}(27 \mathrm{~A})$ & $7679(2)$ & 2972(2) & $7436(2)$ & $60(1)$ \\
\hline
\end{tabular}




\begin{tabular}{lrrrl}
$\mathrm{C}(27 \mathrm{~B})$ & $6795(2)$ & $3855(2)$ & $7109(2)$ & $64(1)$ \\
$\mathrm{C}(28)$ & $5044(2)$ & $1425(2)$ & $8077(2)$ & $41(1)$ \\
$\mathrm{C}(28 \mathrm{~A})$ & $5274(2)$ & $917(2)$ & $8570(2)$ & $70(1)$ \\
$\mathrm{C}(28 \mathrm{~B})$ & $4237(2)$ & $1562(2)$ & $8126(2)$ & $78(1)$ \\
$\mathrm{C}(31)$ & $5041(2)$ & $3096(1)$ & $5477(1)$ & $28(1)$ \\
$\mathrm{C}(32)$ & $5775(2)$ & $3236(2)$ & $5423(2)$ & $40(1)$ \\
$\mathrm{C}(33)$ & $6027(2)$ & $3878(2)$ & $5460(2)$ & $50(1)$ \\
$\mathrm{C}(34)$ & $5543(2)$ & $4390(2)$ & $5534(2)$ & $47(1)$ \\
$\mathrm{C}(35)$ & $4815(2)$ & $4265(2)$ & $5578(2)$ & $42(1)$ \\
$\mathrm{C}(36)$ & $4556(2)$ & $3619(1)$ & $5548(1)$ & $35(1)$ \\
$\mathrm{C}(41)$ & $3818(2)$ & $2176(1)$ & $5617(1)$ & $28(1)$ \\
$\mathrm{C}(42)$ & $3296(2)$ & $2484(2)$ & $5243(2)$ & $39(1)$ \\
$\mathrm{C}(43)$ & $2565(2)$ & $2381(2)$ & $5350(2)$ & $50(1)$ \\
$\mathrm{C}(44)$ & $2347(2)$ & $1957(2)$ & $5815(2)$ & $59(1)$ \\
$\mathrm{C}(45)$ & $2846(2)$ & $1640(2)$ & $6180(2)$ & $53(1)$ \\
$\mathrm{C}(46)$ & $3580(2)$ & $1754(2)$ & $6086(1)$ & $40(1)$ \\
& & & & \\
\hline
\end{tabular}


Table S3. Bond lengths $[\AA]$ and angles $\left[^{\circ}\right]$ for $\mathbf{5} \mathbf{A I C l}_{\mathbf{2}}$.

\begin{tabular}{|c|c|}
\hline $\mathrm{Cl}(1)-\mathrm{Al}$ & $2.1228(12)$ \\
\hline $\mathrm{Cl}(2)-\mathrm{Al}$ & $2.1007(12)$ \\
\hline $\mathrm{P}-\mathrm{C}(3)$ & $1.830(3)$ \\
\hline P-C(41) & $1.831(3)$ \\
\hline P-C $(31)$ & $1.831(3)$ \\
\hline Al-N(1) & $1.861(2)$ \\
\hline Al-N(2) & $1.867(2)$ \\
\hline $\mathrm{N}(1)-\mathrm{C}(2)$ & $1.334(3)$ \\
\hline $\mathrm{N}(1)-\mathrm{C}(11)$ & $1.462(3)$ \\
\hline $\mathrm{N}(2)-\mathrm{C}(4)$ & $1.339(3)$ \\
\hline $\mathrm{N}(2)-\mathrm{C}(21)$ & $1.467(3)$ \\
\hline $\mathrm{C}(1)-\mathrm{C}(2)$ & $1.511(4)$ \\
\hline $\mathrm{C}(2)-\mathrm{C}(3)$ & $1.423(4)$ \\
\hline $\mathrm{C}(3)-\mathrm{C}(4)$ & $1.433(4)$ \\
\hline $\mathrm{C}(4)-\mathrm{C}(5)$ & $1.503(4)$ \\
\hline $\mathrm{C}(11)-\mathrm{C}(12)$ & $1.394(4)$ \\
\hline $\mathrm{C}(11)-\mathrm{C}(16)$ & $1.408(4)$ \\
\hline $\mathrm{C}(12)-\mathrm{C}(13)$ & $1.400(4)$ \\
\hline $\mathrm{C}(12)-\mathrm{C}(17)$ & $1.521(4)$ \\
\hline$C(13)-C(14)$ & $1.367(5)$ \\
\hline$C(14)-C(15)$ & $1.371(5)$ \\
\hline$C(15)-C(16)$ & $1.386(4)$ \\
\hline $\mathrm{C}(16)-\mathrm{C}(18)$ & $1.522(4)$ \\
\hline $\mathrm{C}(17)-\mathrm{C}(17 \mathrm{~A})$ & $1.526(5)$ \\
\hline $\mathrm{C}(17)-\mathrm{C}(17 \mathrm{~B})$ & $1.530(5)$ \\
\hline $\mathrm{C}(18)-\mathrm{C}(18 \mathrm{~A})$ & $1.514(5)$ \\
\hline $\mathrm{C}(18)-\mathrm{C}(18 \mathrm{~B})$ & $1.521(5)$ \\
\hline$C(21)-C(22)$ & $1.399(4)$ \\
\hline$C(21)-C(26)$ & $1.403(4)$ \\
\hline$C(22)-C(23)$ & $1.390(4)$ \\
\hline$C(22)-C(27)$ & $1.525(4)$ \\
\hline$C(23)-C(24)$ & $1.376(5)$ \\
\hline$C(24)-C(25)$ & $1.368(4)$ \\
\hline$C(25)-C(26)$ & $1.393(4)$ \\
\hline
\end{tabular}




\begin{tabular}{|c|c|}
\hline$C(26)-C(28)$ & $1.516(4)$ \\
\hline $\mathrm{C}(27)-\mathrm{C}(27 \mathrm{~A})$ & $1.525(5)$ \\
\hline $\mathrm{C}(27)-\mathrm{C}(27 \mathrm{~B})$ & $1.529(4)$ \\
\hline $\mathrm{C}(28)-\mathrm{C}(28 \mathrm{~B})$ & $1.513(5)$ \\
\hline $\mathrm{C}(28)-\mathrm{C}(28 \mathrm{~A})$ & $1.527(5)$ \\
\hline $\mathrm{C}(31)-\mathrm{C}(32)$ & $1.383(4)$ \\
\hline$C(31)-C(36)$ & $1.394(4)$ \\
\hline$C(32)-C(33)$ & $1.383(4)$ \\
\hline $\mathrm{C}(33)-\mathrm{C}(34)$ & $1.374(5)$ \\
\hline$C(34)-C(35)$ & $1.365(5)$ \\
\hline$C(35)-C(36)$ & $1.393(4)$ \\
\hline$C(41)-C(46)$ & $1.384(4)$ \\
\hline$C(41)-C(42)$ & $1.393(4)$ \\
\hline$C(42)-C(43)$ & $1.378(4)$ \\
\hline$C(43)-C(44)$ & $1.369(5)$ \\
\hline$C(44)-C(45)$ & $1.361(5)$ \\
\hline$C(45)-C(46)$ & $1.383(4)$ \\
\hline C(3)-P-C(41) & $105.68(12)$ \\
\hline C(3)-P-C(31) & $103.36(12)$ \\
\hline C(41)-P-C(31) & $106.50(13)$ \\
\hline $\mathrm{N}(1)-\mathrm{Al}-\mathrm{N}(2)$ & $98.32(10)$ \\
\hline $\mathrm{N}(1)-\mathrm{Al}-\mathrm{Cl}(2)$ & $111.43(8)$ \\
\hline $\mathrm{N}(2)-\mathrm{Al}-\mathrm{Cl}(2)$ & $115.08(8)$ \\
\hline $\mathrm{N}(1)-\mathrm{Al}-\mathrm{Cl}(1)$ & 108.61(8) \\
\hline $\mathrm{N}(2)-\mathrm{Al}-\mathrm{Cl}(1)$ & $110.26(8)$ \\
\hline $\mathrm{Cl}(2)-\mathrm{Al}-\mathrm{Cl}(1)$ & $112.21(6)$ \\
\hline $\mathrm{C}(2)-\mathrm{N}(1)-\mathrm{C}(11)$ & 119.2(2) \\
\hline $\mathrm{C}(2)-\mathrm{N}(1)-\mathrm{Al}$ & $118.22(18)$ \\
\hline $\mathrm{C}(11)-\mathrm{N}(1)-\mathrm{Al}$ & $122.09(17)$ \\
\hline $\mathrm{C}(4)-\mathrm{N}(2)-\mathrm{C}(21)$ & $117.0(2)$ \\
\hline $\mathrm{C}(4)-\mathrm{N}(2)-\mathrm{Al}$ & $118.60(18)$ \\
\hline $\mathrm{C}(21)-\mathrm{N}(2)-\mathrm{Al}$ & $124.23(17)$ \\
\hline $\mathrm{N}(1)-\mathrm{C}(2)-\mathrm{C}(3)$ & $123.5(2)$ \\
\hline $\mathrm{N}(1)-\mathrm{C}(2)-\mathrm{C}(1)$ & $118.7(2)$ \\
\hline$C(3)-C(2)-C(1)$ & $117.8(2)$ \\
\hline
\end{tabular}




\begin{tabular}{|c|c|}
\hline$C(2)-C(3)-C(4)$ & $123.9(2)$ \\
\hline$C(2)-C(3)-P$ & $114.02(19)$ \\
\hline$C(4)-C(3)-P$ & $122.1(2)$ \\
\hline $\mathrm{N}(2)-\mathrm{C}(4)-\mathrm{C}(3)$ & $122.9(2)$ \\
\hline $\mathrm{N}(2)-\mathrm{C}(4)-\mathrm{C}(5)$ & $117.8(2)$ \\
\hline$C(3)-C(4)-C(5)$ & $119.2(2)$ \\
\hline$C(12)-C(11)-C(16)$ & $121.5(3)$ \\
\hline $\mathrm{C}(12)-\mathrm{C}(11)-\mathrm{N}(1)$ & $120.5(3)$ \\
\hline $\mathrm{C}(16)-\mathrm{C}(11)-\mathrm{N}(1)$ & $118.0(2)$ \\
\hline $\mathrm{C}(11)-\mathrm{C}(12)-\mathrm{C}(13)$ & $118.0(3)$ \\
\hline $\mathrm{C}(11)-\mathrm{C}(12)-\mathrm{C}(17)$ & $123.4(3)$ \\
\hline $\mathrm{C}(13)-\mathrm{C}(12)-\mathrm{C}(17)$ & $118.7(3)$ \\
\hline $\mathrm{C}(14)-\mathrm{C}(13)-\mathrm{C}(12)$ & $120.8(3)$ \\
\hline $\mathrm{C}(13)-\mathrm{C}(14)-\mathrm{C}(15)$ & $120.7(3)$ \\
\hline $\mathrm{C}(14)-\mathrm{C}(15)-\mathrm{C}(16)$ & 121.2(3) \\
\hline $\mathrm{C}(15)-\mathrm{C}(16)-\mathrm{C}(11)$ & $117.8(3)$ \\
\hline $\mathrm{C}(15)-\mathrm{C}(16)-\mathrm{C}(18)$ & $120.3(3)$ \\
\hline $\mathrm{C}(11)-\mathrm{C}(16)-\mathrm{C}(18)$ & $121.9(3)$ \\
\hline $\mathrm{C}(12)-\mathrm{C}(17)-\mathrm{C}(17 \mathrm{~A})$ & $110.3(3)$ \\
\hline $\mathrm{C}(12)-\mathrm{C}(17)-\mathrm{C}(17 \mathrm{~B})$ & $112.5(3)$ \\
\hline $\mathrm{C}(17 \mathrm{~A})-\mathrm{C}(17)-\mathrm{C}(17 \mathrm{~B})$ & $110.1(3)$ \\
\hline $\mathrm{C}(18 \mathrm{~A})-\mathrm{C}(18)-\mathrm{C}(18 \mathrm{~B})$ & $110.5(3)$ \\
\hline $\mathrm{C}(18 \mathrm{~A})-\mathrm{C}(18)-\mathrm{C}(16)$ & $112.0(3)$ \\
\hline $\mathrm{C}(18 \mathrm{~B})-\mathrm{C}(18)-\mathrm{C}(16)$ & $112.4(3)$ \\
\hline$C(22)-C(21)-C(26)$ & $121.3(3)$ \\
\hline $\mathrm{C}(22)-\mathrm{C}(21)-\mathrm{N}(2)$ & $119.8(2)$ \\
\hline $\mathrm{C}(26)-\mathrm{C}(21)-\mathrm{N}(2)$ & $118.8(2)$ \\
\hline $\mathrm{C}(23)-\mathrm{C}(22)-\mathrm{C}(21)$ & $117.7(3)$ \\
\hline $\mathrm{C}(23)-\mathrm{C}(22)-\mathrm{C}(27)$ & $118.6(3)$ \\
\hline $\mathrm{C}(21)-\mathrm{C}(22)-\mathrm{C}(27)$ & $123.7(3)$ \\
\hline $\mathrm{C}(24)-\mathrm{C}(23)-\mathrm{C}(22)$ & $121.7(3)$ \\
\hline$C(25)-C(24)-C(23)$ & $119.9(3)$ \\
\hline$C(24)-C(25)-C(26)$ & $121.3(3)$ \\
\hline $\mathrm{C}(25)-\mathrm{C}(26)-\mathrm{C}(21)$ & 118.1(3) \\
\hline$C(25)-C(26)-C(28)$ & $118.4(3)$ \\
\hline $\mathrm{C}(21)-\mathrm{C}(26)-\mathrm{C}(28)$ & $123.5(3)$ \\
\hline
\end{tabular}




\begin{tabular}{ll}
$\mathrm{C}(22)-\mathrm{C}(27)-\mathrm{C}(27 \mathrm{~A})$ & $109.8(3)$ \\
$\mathrm{C}(22)-\mathrm{C}(27)-\mathrm{C}(27 \mathrm{~B})$ & $113.0(3)$ \\
$\mathrm{C}(27 \mathrm{~A})-\mathrm{C}(27)-\mathrm{C}(27 \mathrm{~B})$ & $110.7(3)$ \\
$\mathrm{C}(28 \mathrm{~B})-\mathrm{C}(28)-\mathrm{C}(26)$ & $112.7(3)$ \\
$\mathrm{C}(28 \mathrm{~B})-\mathrm{C}(28)-\mathrm{C}(28 \mathrm{~A})$ & $110.2(3)$ \\
$\mathrm{C}(26)-\mathrm{C}(28)-\mathrm{C}(28 A)$ & $110.5(3)$ \\
$\mathrm{C}(32)-\mathrm{C}(31)-\mathrm{C}(36)$ & $118.5(3)$ \\
$\mathrm{C}(32)-\mathrm{C}(31)-\mathrm{P}$ & $116.4(2)$ \\
$\mathrm{C}(36)-\mathrm{C}(31)-\mathrm{P}$ & $125.0(2)$ \\
$\mathrm{C}(31)-\mathrm{C}(32)-\mathrm{C}(33)$ & $121.0(3)$ \\
$\mathrm{C}(34)-\mathrm{C}(33)-\mathrm{C}(32)$ & $119.9(3)$ \\
$\mathrm{C}(35)-\mathrm{C}(34)-\mathrm{C}(33)$ & $120.2(3)$ \\
$\mathrm{C}(34)-\mathrm{C}(35)-\mathrm{C}(36)$ & $120.4(3)$ \\
$\mathrm{C}(35)-\mathrm{C}(36)-\mathrm{C}(31)$ & $120.0(3)$ \\
$\mathrm{C}(46)-\mathrm{C}(41)-\mathrm{C}(42)$ & $117.9(3)$ \\
$\mathrm{C}(46)-\mathrm{C}(41)-\mathrm{P}$ & $121.3(2)$ \\
$\mathrm{C}(42)-\mathrm{C}(41)-\mathrm{P}$ & $120.0(2)$ \\
$\mathrm{C}(43)-\mathrm{C}(42)-\mathrm{C}(41)$ & $120.7(3)$ \\
$\mathrm{C}(44)-\mathrm{C}(43)-\mathrm{C}(42)$ & $119.9(3)$ \\
$\mathrm{C}(45)-\mathrm{C}(44)-\mathrm{C}(43)$ & $120.6(3)$ \\
$\mathrm{C}(44)-\mathrm{C}(45)-\mathrm{C}(46)$ & $119.8(3)$ \\
$\mathrm{C}(45)-\mathrm{C}(46)-\mathrm{C}(41)$ & $121.0(3)$ \\
\hline
\end{tabular}

Symmetry transformations used to generate equivalent atoms: 
Table S4. Anisotropic displacement parameters $\left(\AA^{2} \mathrm{x} 10^{3}\right)$ for $\mathbf{5} \mathbf{A l C l}_{2}$. The anisotropic displacement factor exponent takes the form: $-2 \pi^{2}\left[h^{2} a^{* 2} U^{11}+\ldots+2 h k a^{*} b^{*} U^{12}\right]$

\begin{tabular}{|c|c|c|c|c|c|c|}
\hline & $\mathrm{U}^{11}$ & $\mathrm{U}^{22}$ & $\mathrm{U}^{33}$ & $\mathrm{U}^{23}$ & $\mathrm{U}^{13}$ & $\mathrm{U}^{12}$ \\
\hline $\mathrm{Cl}(1)$ & $37(1)$ & $59(1)$ & $63(1)$ & $-20(1)$ & $15(1)$ & $-13(1)$ \\
\hline $\mathrm{Cl}(2)$ & $85(1)$ & $48(1)$ & $49(1)$ & $-5(1)$ & $-36(1)$ & $20(1)$ \\
\hline $\mathrm{P}$ & $28(1)$ & $26(1)$ & $23(1)$ & $1(1)$ & $0(1)$ & $4(1)$ \\
\hline $\mathrm{Al}$ & $30(1)$ & $29(1)$ & $28(1)$ & $-5(1)$ & $-6(1)$ & $5(1)$ \\
\hline $\mathrm{N}(1)$ & $30(1)$ & $22(1)$ & $23(1)$ & $0(1)$ & $-2(1)$ & $4(1)$ \\
\hline $\mathrm{N}(2)$ & $29(1)$ & $23(1)$ & $21(1)$ & $-1(1)$ & $0(1)$ & $1(1)$ \\
\hline $\mathrm{C}(1)$ & $35(2)$ & $28(2)$ & $35(2)$ & $-4(1)$ & $-8(1)$ & $1(1)$ \\
\hline $\mathrm{C}(2)$ & $28(2)$ & $23(1)$ & $22(1)$ & $1(1)$ & $4(1)$ & $-3(1)$ \\
\hline $\mathrm{C}(3)$ & $27(2)$ & $22(1)$ & $23(1)$ & $0(1)$ & $2(1)$ & $-1(1)$ \\
\hline$C(4)$ & $29(2)$ & $21(1)$ & $26(2)$ & $0(1)$ & $2(1)$ & $-2(1)$ \\
\hline$C(5)$ & $37(2)$ & $35(2)$ & $26(2)$ & $-3(1)$ & $-1(1)$ & $8(1)$ \\
\hline $\mathrm{C}(11)$ & $34(2)$ & $23(2)$ & $30(2)$ & $-2(1)$ & $-8(1)$ & $4(1)$ \\
\hline $\mathrm{C}(12)$ & $37(2)$ & $30(2)$ & $33(2)$ & $-3(1)$ & $-9(1)$ & $9(1)$ \\
\hline $\mathrm{C}(13)$ & $56(2)$ & $33(2)$ & $38(2)$ & $-13(2)$ & $-9(2)$ & $16(2)$ \\
\hline$C(14)$ & $75(3)$ & $21(2)$ & $49(2)$ & $-6(2)$ & $-15(2)$ & $10(2)$ \\
\hline $\mathrm{C}(15)$ & $69(2)$ & $24(2)$ & $43(2)$ & $3(1)$ & $-11(2)$ & $-3(2)$ \\
\hline$C(16)$ & $50(2)$ & $23(2)$ & $33(2)$ & $-1(1)$ & $-7(1)$ & $-3(1)$ \\
\hline $\mathrm{C}(17)$ & $47(2)$ & $34(2)$ & $38(2)$ & $-2(1)$ & $8(2)$ & $15(2)$ \\
\hline $\mathrm{C}(17 \mathrm{~A})$ & $52(2)$ & $51(2)$ & $88(3)$ & $1(2)$ & $21(2)$ & $14(2)$ \\
\hline $\mathrm{C}(17 \mathrm{~B})$ & $106(4)$ & $59(2)$ & $38(2)$ & $3(2)$ & $10(2)$ & $27(2)$ \\
\hline $\mathrm{C}(18)$ & 74(3) & $29(2)$ & $39(2)$ & $1(2)$ & $7(2)$ & $-10(2)$ \\
\hline $\mathrm{C}(18 \mathrm{~A})$ & $74(3)$ & $68(3)$ & $70(3)$ & $-5(2)$ & $26(2)$ & $-11(2)$ \\
\hline $\mathrm{C}(18 \mathrm{~B})$ & $148(5)$ & $50(2)$ & $47(2)$ & $17(2)$ & $20(3)$ & $-7(3)$ \\
\hline $\mathrm{C}(21)$ & $30(2)$ & $28(2)$ & $23(1)$ & $-3(1)$ & $-3(1)$ & $5(1)$ \\
\hline $\mathrm{C}(22)$ & $40(2)$ & $31(2)$ & $30(2)$ & $-5(1)$ & $-2(1)$ & $2(1)$ \\
\hline $\mathrm{C}(23)$ & $54(2)$ & $35(2)$ & $41(2)$ & $-11(2)$ & $-4(2)$ & $-3(2)$ \\
\hline $\mathrm{C}(24)$ & $58(2)$ & $44(2)$ & $29(2)$ & $-13(2)$ & $-3(2)$ & $6(2)$ \\
\hline $\mathrm{C}(25)$ & $42(2)$ & $45(2)$ & $27(2)$ & $-2(1)$ & $5(1)$ & $10(2)$ \\
\hline $\mathrm{C}(26)$ & $33(2)$ & $33(2)$ & $26(2)$ & $0(1)$ & $-2(1)$ & $5(1)$ \\
\hline $\mathrm{C}(27)$ & $49(2)$ & $32(2)$ & $43(2)$ & $-6(2)$ & $5(2)$ & $-11(2)$ \\
\hline $\mathrm{C}(27 \mathrm{~A})$ & $51(2)$ & $67(3)$ & $62(2)$ & $-11(2)$ & $8(2)$ & $-19(2)$ \\
\hline
\end{tabular}




\begin{tabular}{lllllll}
$\mathrm{C}(27 \mathrm{~B})$ & $92(3)$ & $40(2)$ & $59(3)$ & $0(2)$ & $17(2)$ & $-13(2)$ \\
$\mathrm{C}(28)$ & $47(2)$ & $44(2)$ & $33(2)$ & $0(2)$ & $11(2)$ & $-8(2)$ \\
$\mathrm{C}(28 \mathrm{~A})$ & $103(4)$ & $48(2)$ & $59(3)$ & $14(2)$ & $7(2)$ & $-11(2)$ \\
$\mathrm{C}(28 \mathrm{~B})$ & $48(3)$ & $85(3)$ & $102(4)$ & $-3(3)$ & $13(2)$ & $-20(2)$ \\
$\mathrm{C}(31)$ & $34(2)$ & $26(2)$ & $24(2)$ & $2(1)$ & $2(1)$ & $1(1)$ \\
$\mathrm{C}(32)$ & $38(2)$ & $32(2)$ & $51(2)$ & $4(2)$ & $13(2)$ & $3(1)$ \\
$\mathrm{C}(33)$ & $40(2)$ & $41(2)$ & $69(3)$ & $7(2)$ & $11(2)$ & $-10(2)$ \\
$\mathrm{C}(34)$ & $58(2)$ & $29(2)$ & $54(2)$ & $4(2)$ & $4(2)$ & $-10(2)$ \\
$\mathrm{C}(35)$ & $53(2)$ & $27(2)$ & $46(2)$ & $0(1)$ & $-2(2)$ & $7(2)$ \\
$\mathrm{C}(36)$ & $35(2)$ & $31(2)$ & $40(2)$ & $2(1)$ & $-3(1)$ & $4(1)$ \\
$\mathrm{C}(41)$ & $27(2)$ & $29(2)$ & $28(2)$ & $-4(1)$ & $2(1)$ & $1(1)$ \\
$\mathrm{C}(42)$ & $37(2)$ & $37(2)$ & $42(2)$ & $-1(1)$ & $-7(1)$ & $4(2)$ \\
$\mathrm{C}(43)$ & $31(2)$ & $52(2)$ & $68(3)$ & $-8(2)$ & $-10(2)$ & $6(2)$ \\
$\mathrm{C}(44)$ & $31(2)$ & $65(3)$ & $79(3)$ & $-12(2)$ & $14(2)$ & $-6(2)$ \\
$\mathrm{C}(45)$ & $47(2)$ & $53(2)$ & $57(2)$ & $4(2)$ & $21(2)$ & $-6(2)$ \\
$\mathrm{C}(46)$ & $38(2)$ & $43(2)$ & $38(2)$ & $1(2)$ & $5(1)$ & $0(2)$ \\
& & & & & & \\
\hline
\end{tabular}


Table S5. Torsion angles $\left[{ }^{\circ}\right]$ for $\mathbf{5} \mathbf{A l C l}_{\mathbf{2}}$.

\begin{tabular}{|c|c|}
\hline $\mathrm{N}(2)-\mathrm{Al}-\mathrm{N}(1)-\mathrm{C}(2)$ & $35.9(2)$ \\
\hline $\mathrm{Cl}(2)-\mathrm{Al}-\mathrm{N}(1)-\mathrm{C}(2)$ & $157.07(18)$ \\
\hline $\mathrm{Cl}(1)-\mathrm{Al}-\mathrm{N}(1)-\mathrm{C}(2)$ & $-78.8(2)$ \\
\hline $\mathrm{N}(2)-\mathrm{Al}-\mathrm{N}(1)-\mathrm{C}(11)$ & $-152.5(2)$ \\
\hline $\mathrm{Cl}(2)-\mathrm{Al}-\mathrm{N}(1)-\mathrm{C}(11)$ & $-31.3(2)$ \\
\hline $\mathrm{Cl}(1)-\mathrm{Al}-\mathrm{N}(1)-\mathrm{C}(11)$ & $92.8(2)$ \\
\hline $\mathrm{N}(1)-\mathrm{Al}-\mathrm{N}(2)-\mathrm{C}(4)$ & $-33.6(2)$ \\
\hline $\mathrm{Cl}(2)-\mathrm{Al}-\mathrm{N}(2)-\mathrm{C}(4)$ & $-152.08(18)$ \\
\hline $\mathrm{Cl}(1)-\mathrm{Al}-\mathrm{N}(2)-\mathrm{C}(4)$ & $79.8(2)$ \\
\hline $\mathrm{N}(1)-\mathrm{Al}-\mathrm{N}(2)-\mathrm{C}(21)$ & $151.1(2)$ \\
\hline $\mathrm{Cl}(2)-\mathrm{Al}-\mathrm{N}(2)-\mathrm{C}(21)$ & $32.7(2)$ \\
\hline $\mathrm{Cl}(1)-\mathrm{Al}-\mathrm{N}(2)-\mathrm{C}(21)$ & $-95.5(2)$ \\
\hline $\mathrm{C}(11)-\mathrm{N}(1)-\mathrm{C}(2)-\mathrm{C}(3)$ & $172.9(2)$ \\
\hline $\mathrm{Al}-\mathrm{N}(1)-\mathrm{C}(2)-\mathrm{C}(3)$ & $-15.2(3)$ \\
\hline $\mathrm{C}(11)-\mathrm{N}(1)-\mathrm{C}(2)-\mathrm{C}(1)$ & $-3.9(4)$ \\
\hline $\mathrm{Al}-\mathrm{N}(1)-\mathrm{C}(2)-\mathrm{C}(1)$ & $167.95(19)$ \\
\hline $\mathrm{N}(1)-\mathrm{C}(2)-\mathrm{C}(3)-\mathrm{C}(4)$ & $-18.3(4)$ \\
\hline $\mathrm{C}(1)-\mathrm{C}(2)-\mathrm{C}(3)-\mathrm{C}(4)$ & $158.6(3)$ \\
\hline $\mathrm{N}(1)-\mathrm{C}(2)-\mathrm{C}(3)-\mathrm{P}$ & $162.4(2)$ \\
\hline $\mathrm{C}(1)-\mathrm{C}(2)-\mathrm{C}(3)-\mathrm{P}$ & $-20.8(3)$ \\
\hline $\mathrm{C}(41)-\mathrm{P}-\mathrm{C}(3)-\mathrm{C}(2)$ & $102.1(2)$ \\
\hline $\mathrm{C}(31)-\mathrm{P}-\mathrm{C}(3)-\mathrm{C}(2)$ & $-146.1(2)$ \\
\hline $\mathrm{C}(41)-\mathrm{P}-\mathrm{C}(3)-\mathrm{C}(4)$ & $-77.2(2)$ \\
\hline $\mathrm{C}(31)-\mathrm{P}-\mathrm{C}(3)-\mathrm{C}(4)$ & $34.5(2)$ \\
\hline $\mathrm{C}(21)-\mathrm{N}(2)-\mathrm{C}(4)-\mathrm{C}(3)$ & $-173.8(2)$ \\
\hline $\mathrm{Al}-\mathrm{N}(2)-\mathrm{C}(4)-\mathrm{C}(3)$ & $10.6(3)$ \\
\hline $\mathrm{C}(21)-\mathrm{N}(2)-\mathrm{C}(4)-\mathrm{C}(5)$ & $5.0(4)$ \\
\hline $\mathrm{Al}-\mathrm{N}(2)-\mathrm{C}(4)-\mathrm{C}(5)$ & $-170.60(19)$ \\
\hline $\mathrm{C}(2)-\mathrm{C}(3)-\mathrm{C}(4)-\mathrm{N}(2)$ & $20.9(4)$ \\
\hline $\mathrm{P}-\mathrm{C}(3)-\mathrm{C}(4)-\mathrm{N}(2)$ & $-159.8(2)$ \\
\hline$C(2)-C(3)-C(4)-C(5)$ & $-157.9(3)$ \\
\hline $\mathrm{P}-\mathrm{C}(3)-\mathrm{C}(4)-\mathrm{C}(5)$ & $21.4(3)$ \\
\hline $\mathrm{C}(2)-\mathrm{N}(1)-\mathrm{C}(11)-\mathrm{C}(12)$ & $82.9(3)$ \\
\hline $\mathrm{Al}-\mathrm{N}(1)-\mathrm{C}(11)-\mathrm{C}(12)$ & $-88.7(3)$ \\
\hline
\end{tabular}




\begin{tabular}{|c|c|}
\hline $\mathrm{C}(2)-\mathrm{N}(1)-\mathrm{C}(11)-\mathrm{C}(16)$ & $-98.6(3)$ \\
\hline $\mathrm{Al}-\mathrm{N}(1)-\mathrm{C}(11)-\mathrm{C}(16)$ & $89.9(3)$ \\
\hline$C(16)-C(11)-C(12)-C(13)$ & $2.5(4)$ \\
\hline $\mathrm{N}(1)-\mathrm{C}(11)-\mathrm{C}(12)-\mathrm{C}(13)$ & $-179.0(3)$ \\
\hline$C(16)-C(11)-C(12)-C(17)$ & $-177.8(3)$ \\
\hline $\mathrm{N}(1)-\mathrm{C}(11)-\mathrm{C}(12)-\mathrm{C}(17)$ & $0.7(4)$ \\
\hline $\mathrm{C}(11)-\mathrm{C}(12)-\mathrm{C}(13)-\mathrm{C}(14)$ & $-0.5(5)$ \\
\hline $\mathrm{C}(17)-\mathrm{C}(12)-\mathrm{C}(13)-\mathrm{C}(14)$ & $179.8(3)$ \\
\hline$C(12)-C(13)-C(14)-C(15)$ & $-0.5(5)$ \\
\hline $\mathrm{C}(13)-\mathrm{C}(14)-\mathrm{C}(15)-\mathrm{C}(16)$ & $-0.5(5)$ \\
\hline$C(14)-C(15)-C(16)-C(11)$ & $2.4(5)$ \\
\hline$C(14)-C(15)-C(16)-C(18)$ & $-179.4(3)$ \\
\hline $\mathrm{C}(12)-\mathrm{C}(11)-\mathrm{C}(16)-\mathrm{C}(15)$ & $-3.4(4)$ \\
\hline $\mathrm{N}(1)-\mathrm{C}(11)-\mathrm{C}(16)-\mathrm{C}(15)$ & $178.0(3)$ \\
\hline $\mathrm{C}(12)-\mathrm{C}(11)-\mathrm{C}(16)-\mathrm{C}(18)$ & $178.4(3)$ \\
\hline $\mathrm{N}(1)-\mathrm{C}(11)-\mathrm{C}(16)-\mathrm{C}(18)$ & $-0.2(4)$ \\
\hline $\mathrm{C}(11)-\mathrm{C}(12)-\mathrm{C}(17)-\mathrm{C}(17 \mathrm{~A})$ & $112.5(3)$ \\
\hline $\mathrm{C}(13)-\mathrm{C}(12)-\mathrm{C}(17)-\mathrm{C}(17 \mathrm{~A})$ & $-67.8(4)$ \\
\hline $\mathrm{C}(11)-\mathrm{C}(12)-\mathrm{C}(17)-\mathrm{C}(17 \mathrm{~B})$ & $-124.1(3)$ \\
\hline $\mathrm{C}(13)-\mathrm{C}(12)-\mathrm{C}(17)-\mathrm{C}(17 \mathrm{~B})$ & $55.6(4)$ \\
\hline $\mathrm{C}(15)-\mathrm{C}(16)-\mathrm{C}(18)-\mathrm{C}(18 \mathrm{~A})$ & $-91.9(4)$ \\
\hline $\mathrm{C}(11)-\mathrm{C}(16)-\mathrm{C}(18)-\mathrm{C}(18 \mathrm{~A})$ & $86.3(4)$ \\
\hline $\mathrm{C}(15)-\mathrm{C}(16)-\mathrm{C}(18)-\mathrm{C}(18 \mathrm{~B})$ & $33.3(5)$ \\
\hline $\mathrm{C}(11)-\mathrm{C}(16)-\mathrm{C}(18)-\mathrm{C}(18 \mathrm{~B})$ & $-148.6(3)$ \\
\hline $\mathrm{C}(4)-\mathrm{N}(2)-\mathrm{C}(21)-\mathrm{C}(22)$ & $-92.2(3)$ \\
\hline $\mathrm{Al}-\mathrm{N}(2)-\mathrm{C}(21)-\mathrm{C}(22)$ & $83.2(3)$ \\
\hline $\mathrm{C}(4)-\mathrm{N}(2)-\mathrm{C}(21)-\mathrm{C}(26)$ & $84.6(3)$ \\
\hline $\mathrm{Al}-\mathrm{N}(2)-\mathrm{C}(21)-\mathrm{C}(26)$ & $-100.1(3)$ \\
\hline$C(26)-C(21)-C(22)-C(23)$ & $-2.3(4)$ \\
\hline $\mathrm{N}(2)-\mathrm{C}(21)-\mathrm{C}(22)-\mathrm{C}(23)$ & $174.3(3)$ \\
\hline $\mathrm{C}(26)-\mathrm{C}(21)-\mathrm{C}(22)-\mathrm{C}(27)$ & $176.6(3)$ \\
\hline $\mathrm{N}(2)-\mathrm{C}(21)-\mathrm{C}(22)-\mathrm{C}(27)$ & $-6.8(4)$ \\
\hline $\mathrm{C}(21)-\mathrm{C}(22)-\mathrm{C}(23)-\mathrm{C}(24)$ & $-0.1(5)$ \\
\hline $\mathrm{C}(27)-\mathrm{C}(22)-\mathrm{C}(23)-\mathrm{C}(24)$ & $-179.0(3)$ \\
\hline $\mathrm{C}(22)-\mathrm{C}(23)-\mathrm{C}(24)-\mathrm{C}(25)$ & $1.4(5)$ \\
\hline$C(23)-C(24)-C(25)-C(26)$ & $-0.3(5)$ \\
\hline
\end{tabular}




\begin{tabular}{|c|c|}
\hline$C(24)-C(25)-C(26)-C(21)$ & $-2.1(4)$ \\
\hline$C(24)-C(25)-C(26)-C(28)$ & $179.3(3)$ \\
\hline$C(22)-C(21)-C(26)-C(25)$ & $3.4(4)$ \\
\hline $\mathrm{N}(2)-\mathrm{C}(21)-\mathrm{C}(26)-\mathrm{C}(25)$ & $-173.3(2)$ \\
\hline $\mathrm{C}(22)-\mathrm{C}(21)-\mathrm{C}(26)-\mathrm{C}(28)$ & $-178.1(3)$ \\
\hline $\mathrm{N}(2)-\mathrm{C}(21)-\mathrm{C}(26)-\mathrm{C}(28)$ & $5.3(4)$ \\
\hline $\mathrm{C}(23)-\mathrm{C}(22)-\mathrm{C}(27)-\mathrm{C}(27 \mathrm{~A})$ & $72.6(4)$ \\
\hline $\mathrm{C}(21)-\mathrm{C}(22)-\mathrm{C}(27)-\mathrm{C}(27 \mathrm{~A})$ & $-106.3(3)$ \\
\hline $\mathrm{C}(23)-\mathrm{C}(22)-\mathrm{C}(27)-\mathrm{C}(27 \mathrm{~B})$ & $-51.6(4)$ \\
\hline $\mathrm{C}(21)-\mathrm{C}(22)-\mathrm{C}(27)-\mathrm{C}(27 \mathrm{~B})$ & $129.6(3)$ \\
\hline $\mathrm{C}(25)-\mathrm{C}(26)-\mathrm{C}(28)-\mathrm{C}(28 \mathrm{~B})$ & $61.8(4)$ \\
\hline $\mathrm{C}(21)-\mathrm{C}(26)-\mathrm{C}(28)-\mathrm{C}(28 \mathrm{~B})$ & $-116.7(3)$ \\
\hline$C(25)-C(26)-C(28)-C(28 A)$ & $-62.0(4)$ \\
\hline $\mathrm{C}(21)-\mathrm{C}(26)-\mathrm{C}(28)-\mathrm{C}(28 \mathrm{~A})$ & $119.5(3)$ \\
\hline C(3)-P-C(31)-C(32) & $60.0(3)$ \\
\hline C(41)-P-C(31)-C(32) & 171.1(2) \\
\hline C(3)-P-C(31)-C(36) & $-123.8(2)$ \\
\hline C(41)-P-C(31)-C(36) & $-12.7(3)$ \\
\hline $\mathrm{C}(36)-\mathrm{C}(31)-\mathrm{C}(32)-\mathrm{C}(33)$ & $2.1(5)$ \\
\hline $\mathrm{P}-\mathrm{C}(31)-\mathrm{C}(32)-\mathrm{C}(33)$ & $178.6(3)$ \\
\hline $\mathrm{C}(31)-\mathrm{C}(32)-\mathrm{C}(33)-\mathrm{C}(34)$ & $-1.7(5)$ \\
\hline $\mathrm{C}(32)-\mathrm{C}(33)-\mathrm{C}(34)-\mathrm{C}(35)$ & $0.7(5)$ \\
\hline $\mathrm{C}(33)-\mathrm{C}(34)-\mathrm{C}(35)-\mathrm{C}(36)$ & $-0.2(5)$ \\
\hline $\mathrm{C}(34)-\mathrm{C}(35)-\mathrm{C}(36)-\mathrm{C}(31)$ & $0.6(5)$ \\
\hline $\mathrm{C}(32)-\mathrm{C}(31)-\mathrm{C}(36)-\mathrm{C}(35)$ & $-1.5(4)$ \\
\hline $\mathrm{P}-\mathrm{C}(31)-\mathrm{C}(36)-\mathrm{C}(35)$ & $-177.7(2)$ \\
\hline C(3)-P-C(41)-C(46) & $-15.2(3)$ \\
\hline C(31)-P-C(41)-C(46) & $-124.6(2)$ \\
\hline C(3)-P-C(41)-C(42) & $175.2(2)$ \\
\hline C(31)-P-C(41)-C(42) & $65.7(3)$ \\
\hline $\mathrm{C}(46)-\mathrm{C}(41)-\mathrm{C}(42)-\mathrm{C}(43)$ & $1.5(5)$ \\
\hline $\mathrm{P}-\mathrm{C}(41)-\mathrm{C}(42)-\mathrm{C}(43)$ & $171.5(2)$ \\
\hline $\mathrm{C}(41)-\mathrm{C}(42)-\mathrm{C}(43)-\mathrm{C}(44)$ & $-1.9(5)$ \\
\hline $\mathrm{C}(42)-\mathrm{C}(43)-\mathrm{C}(44)-\mathrm{C}(45)$ & $0.7(6)$ \\
\hline $\mathrm{C}(43)-\mathrm{C}(44)-\mathrm{C}(45)-\mathrm{C}(46)$ & $1.0(6)$ \\
\hline $\mathrm{C}(44)-\mathrm{C}(45)-\mathrm{C}(46)-\mathrm{C}(41)$ & $-1.4(5)$ \\
\hline
\end{tabular}


C(42)-C(41)-C(46)-C(45)

$0.1(5)$

P-C(41)-C(46)-C(45)

$-169.7(3)$ 


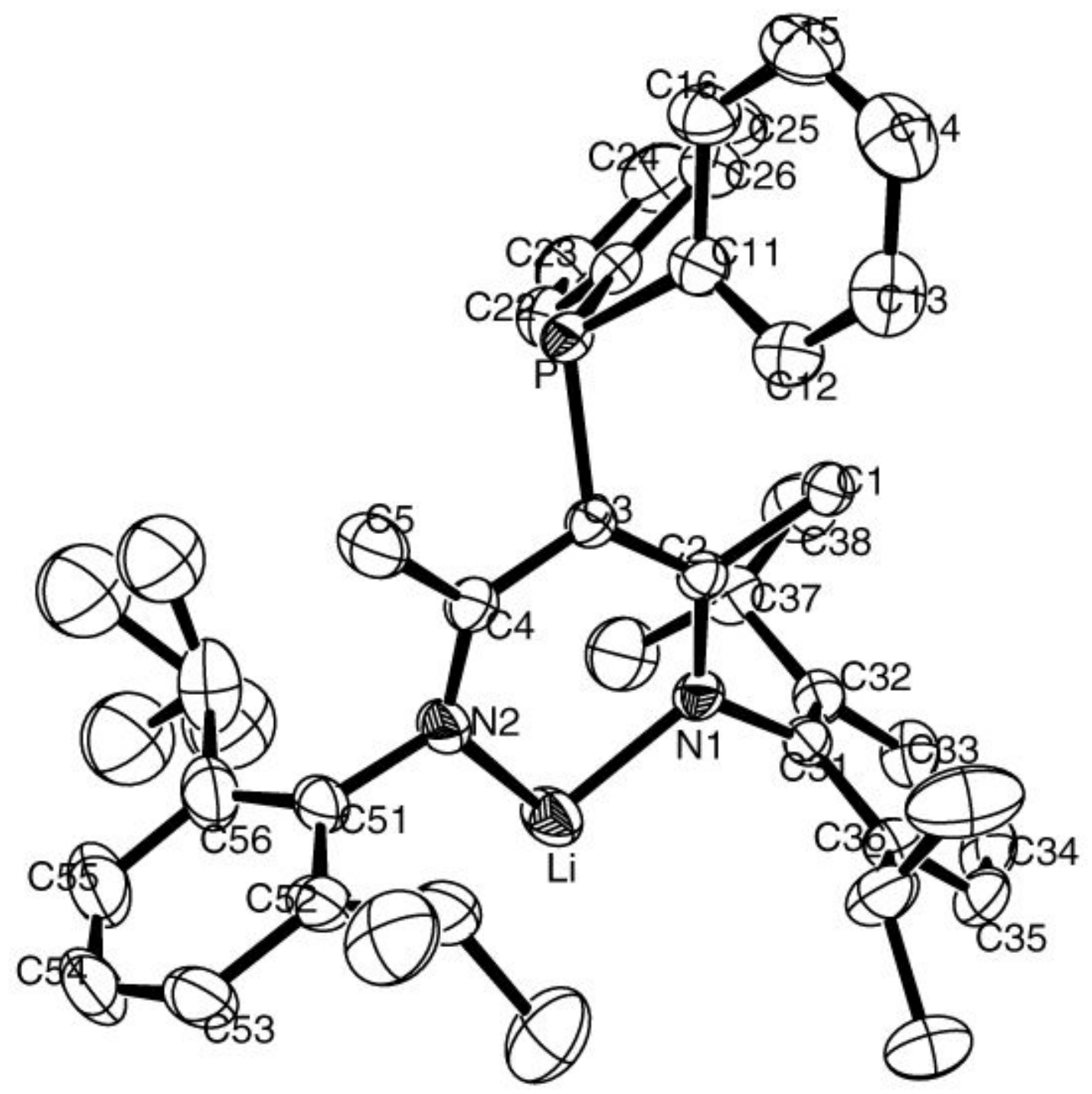

Figure S2: Solid state structure of $\mathbf{5 L i}$. Ellipsoids are 50\% probability, hydrogen atoms and toluene solvate are removed for clarity. Numbering scheme of core atoms is included. 
Table S6. Crystal data and structure refinement for $\mathbf{5} \mathbf{L i}$.

Identification code

Empirical formula

Formula weight

Temperature

Wavelength

Crystal system

Space group

Unit cell dimensions

Volume

Z

Density (calculated)

Absorption coefficient

$\mathrm{F}(000)$

Crystal size

Theta range for data collection

Index ranges

Reflections collected

Independent reflections

Completeness to theta $=26.39^{\circ}$

Absorption correction

Max. and min. transmission

Refinement method

Data / restraints / parameters

Goodness-of-fit on $\mathrm{F}^{2}$

Final $\mathrm{R}$ indices [I $>2 \operatorname{sigma}(\mathrm{I})]$

$\mathrm{R}$ indices (all data)

Largest diff. peak and hole dal0348

C48 H58 Li N2 P

700.87

193(2) K

$0.71073 \AA$

Triclinic

P-1

$\mathrm{a}=10.1138(4) \AA$

$\alpha=77.1930(10)^{\circ}$.

$\mathrm{b}=11.1175(5) \AA$

$\beta=84.7970(10)^{\circ}$.

$\mathrm{c}=19.6442(9) \AA$

$\gamma=78.2080(10)^{\circ}$.

2

$1.105 \mathrm{Mg} / \mathrm{m}^{3}$

$0.099 \mathrm{~mm}^{-1}$

756

$0.43 \times 0.27 \times 0.26 \mathrm{~mm}^{3}$

1.91 to $26.39^{\circ}$.

$-12<=\mathrm{h}<=12,-11<=\mathrm{k}<=13,-24<=\mathrm{l}<=24$

12066

$8468[\mathrm{R}($ int $)=0.0180]$

$98.1 \%$

Integration

0.9748 and 0.9588

Full-matrix least-squares on $\mathrm{F}^{2}$

8468 / 0 / 470

1.021

$\mathrm{R} 1=0.0474, \mathrm{wR} 2=0.1217$

$\mathrm{R} 1=0.0612, \mathrm{wR} 2=0.1312$

0.332 and -0.226 e. $\AA^{-3}$ 
Table S7: Atomic coordinates ( $\mathrm{x} 10^{4}$ ) and equivalent isotropic displacement parameters $\left(\AA^{2} \times 10^{3}\right)$ for $\mathbf{5} \mathbf{L i}$. $\mathbf{U}(\mathrm{eq})$ is defined as one third of the trace of the orthogonalized $\mathrm{U}^{\mathrm{ij}}$ tensor.

\begin{tabular}{|c|c|c|c|c|}
\hline & $\mathrm{x}$ & $\mathrm{y}$ & $\mathrm{z}$ & $\mathrm{U}(\mathrm{eq})$ \\
\hline $\mathrm{P}$ & $-60(1)$ & $3726(1)$ & $3262(1)$ & $28(1)$ \\
\hline $\mathrm{N}(1)$ & $1218(1)$ & $300(1)$ & $2780(1)$ & $27(1)$ \\
\hline $\mathrm{N}(2)$ & $1558(1)$ & $2278(1)$ & 1651(1) & $32(1)$ \\
\hline $\mathrm{C}(1)$ & $-167(2)$ & $825(1)$ & $3796(1)$ & $29(1)$ \\
\hline $\mathrm{C}(2)$ & $526(1)$ & 1195(1) & $3083(1)$ & $25(1)$ \\
\hline $\mathrm{C}(3)$ & $369(2)$ & $2509(1)$ & $2759(1)$ & $27(1)$ \\
\hline $\mathrm{C}(4)$ & $785(2)$ & 2977(1) & 2043(1) & $31(1)$ \\
\hline$C(5)$ & $241(2)$ & $4346(2)$ & $1723(1)$ & $53(1)$ \\
\hline $\mathrm{C}(11)$ & $-1844(2)$ & $3900(1)$ & $3555(1)$ & $32(1)$ \\
\hline $\mathrm{C}(12)$ & $-2752(2)$ & $3340(2)$ & 3301(1) & $37(1)$ \\
\hline $\mathrm{C}(13)$ & $-4121(2)$ & $3584(2)$ & $3490(1)$ & $47(1)$ \\
\hline $\mathrm{C}(14)$ & $-4609(2)$ & 4392(2) & $3936(1)$ & $51(1)$ \\
\hline$C(15)$ & $-3731(2)$ & $4980(2)$ & $4179(1)$ & $49(1)$ \\
\hline$C(16)$ & $-2366(2)$ & $4753(2)$ & $3988(1)$ & $40(1)$ \\
\hline $\mathrm{C}(21)$ & $877(2)$ & $3065(1)$ & $4057(1)$ & $30(1)$ \\
\hline $\mathrm{C}(22)$ & $2277(2)$ & $2721(2)$ & 3961(1) & $39(1)$ \\
\hline $\mathrm{C}(23)$ & $3092(2)$ & $2255(2)$ & $4525(1)$ & $49(1)$ \\
\hline $\mathrm{C}(24)$ & $2529(2)$ & $2143(2)$ & $5199(1)$ & $51(1)$ \\
\hline $\mathrm{C}(25)$ & $1150(2)$ & $2483(2)$ & $5305(1)$ & $48(1)$ \\
\hline$C(26)$ & $331(2)$ & $2946(2)$ & $4739(1)$ & $38(1)$ \\
\hline $\mathrm{C}(31)$ & $1325(2)$ & $-997(1)$ & $3112(1)$ & $28(1)$ \\
\hline $\mathrm{C}(32)$ & $2267(2)$ & $-1552(1)$ & $3629(1)$ & $31(1)$ \\
\hline $\mathrm{C}(33)$ & $2453(2)$ & $-2849(2)$ & $3877(1)$ & $41(1)$ \\
\hline$C(34)$ & $1750(2)$ & $-3578(2)$ & $3624(1)$ & $46(1)$ \\
\hline $\mathrm{C}(35)$ & $822(2)$ & $-3023(2)$ & $3118(1)$ & $42(1)$ \\
\hline$C(36)$ & $591(2)$ & $-1737(2)$ & $2856(1)$ & $34(1)$ \\
\hline $\mathrm{C}(37)$ & $3090(2)$ & $-776(2)$ & $3904(1)$ & $37(1)$ \\
\hline $\mathrm{C}(38)$ & $3283(3)$ & $-1191(2)$ & $4686(1)$ & $63(1)$ \\
\hline $\mathrm{C}(39)$ & $4459(2)$ & $-784(3)$ & $3508(1)$ & $66(1)$ \\
\hline$C(40)$ & $-456(2)$ & $-1123(2)$ & 2311(1) & $47(1)$ \\
\hline $\mathrm{C}(41)$ & $-1799(2)$ & $-583(3)$ & $2642(1)$ & $74(1)$ \\
\hline
\end{tabular}




\begin{tabular}{|c|c|c|c|c|}
\hline $\mathrm{C}(42)$ & $-679(3)$ & $-1994(2)$ & 1845(1) & $64(1)$ \\
\hline $\mathrm{C}(51)$ & 1975(2) & $2790(2)$ & $950(1)$ & $38(1)$ \\
\hline $\mathrm{C}(52)$ & $1172(2)$ & $2846(2)$ & $392(1)$ & $42(1)$ \\
\hline $\mathrm{C}(53)$ & 1751(3) & $3143(2)$ & $-287(1)$ & $57(1)$ \\
\hline $\mathrm{C}(54)$ & $3040(3)$ & $3395(2)$ & $-403(1)$ & $68(1)$ \\
\hline $\mathrm{C}(55)$ & $3786(2)$ & $3389(2)$ & $146(1)$ & $63(1)$ \\
\hline$C(56)$ & $3277(2)$ & $3094(2)$ & $832(1)$ & $50(1)$ \\
\hline $\mathrm{C}(57)$ & $-255(2)$ & $2583(2)$ & $515(1)$ & $46(1)$ \\
\hline $\mathrm{C}(58)$ & $-292(3)$ & $1251(2)$ & $458(1)$ & $67(1)$ \\
\hline $\mathrm{C}(59)$ & $-1246(3)$ & $3495(2)$ & $13(1)$ & $67(1)$ \\
\hline$C(60)$ & $4095(2)$ & $3139(2)$ & $1436(1)$ & $65(1)$ \\
\hline$C(61 \mathrm{~A})$ & $3718(6)$ & $4307(5)$ & $1655(3)$ & $77(1)$ \\
\hline$C(62 A)$ & $5655(6)$ & $2847(6)$ & $1218(3)$ & $95(2)$ \\
\hline$C(61 B)$ & $4477(8)$ & $4467(7)$ & $1376(4)$ & $90(2)$ \\
\hline$C(62 B)$ & $5310(8)$ & 2181(7) & 1641(4) & $96(2)$ \\
\hline $\mathrm{Li}$ & $2254(3)$ & $521(3)$ & 1921(1) & $41(1)$ \\
\hline$C(10 S)$ & $5412(4)$ & $-3141(3)$ & $2387(1)$ & $102(1)$ \\
\hline$C(11 S)$ & $4667(3)$ & $-2138(2)$ & 1837(1) & $60(1)$ \\
\hline$C(12 S)$ & $3414(3)$ & $-2183(2)$ & $1657(2)$ & $77(1)$ \\
\hline$C(13 S)$ & 2761(3) & $-1283(3)$ & $1136(2)$ & $83(1)$ \\
\hline$C(14 S)$ & $3347(3)$ & $-317(2)$ & $781(1)$ & $67(1)$ \\
\hline$C(15 S)$ & $4574(3)$ & $-238(2)$ & $957(2)$ & $78(1)$ \\
\hline$C(16 S)$ & $5235(3)$ & $-1139(3)$ & $1482(2)$ & $82(1)$ \\
\hline
\end{tabular}


Table S8. Bond lengths $[\AA]$ and angles $\left[{ }^{\circ}\right]$ for $\mathbf{5} \mathbf{L i}$.

\begin{tabular}{|c|c|}
\hline $\mathrm{P}-\mathrm{C}(3)$ & $1.8084(15)$ \\
\hline $\mathrm{P}-\mathrm{C}(11)$ & $1.8282(17)$ \\
\hline $\mathrm{P}-\mathrm{C}(21)$ & $1.8309(16)$ \\
\hline $\mathrm{N}(1)-\mathrm{C}(2)$ & $1.3180(18)$ \\
\hline $\mathrm{N}(1)-\mathrm{C}(31)$ & $1.4322(18)$ \\
\hline $\mathrm{N}(1)-\mathrm{Li}$ & $1.899(3)$ \\
\hline $\mathrm{N}(2)-\mathrm{C}(4)$ & $1.310(2)$ \\
\hline $\mathrm{N}(2)-\mathrm{C}(51)$ & $1.4299(19)$ \\
\hline $\mathrm{N}(2)-\mathrm{Li}$ & $1.908(3)$ \\
\hline $\mathrm{C}(1)-\mathrm{C}(2)$ & $1.518(2)$ \\
\hline $\mathrm{C}(2)-\mathrm{C}(3)$ & $1.442(2)$ \\
\hline $\mathrm{C}(3)-\mathrm{C}(4)$ & $1.442(2)$ \\
\hline $\mathrm{C}(4)-\mathrm{C}(5)$ & $1.520(2)$ \\
\hline $\mathrm{C}(11)-\mathrm{C}(12)$ & $1.391(2)$ \\
\hline$C(11)-C(16)$ & $1.405(2)$ \\
\hline$C(12)-C(13)$ & $1.388(2)$ \\
\hline$C(13)-C(14)$ & $1.381(3)$ \\
\hline $\mathrm{C}(14)-\mathrm{C}(15)$ & $1.376(3)$ \\
\hline$C(15)-C(16)$ & $1.384(3)$ \\
\hline$C(21)-C(26)$ & $1.391(2)$ \\
\hline$C(21)-C(22)$ & $1.396(2)$ \\
\hline$C(22)-C(23)$ & $1.383(3)$ \\
\hline$C(23)-C(24)$ & $1.382(3)$ \\
\hline$C(24)-C(25)$ & $1.378(3)$ \\
\hline$C(25)-C(26)$ & $1.388(2)$ \\
\hline $\mathrm{C}(31)-\mathrm{C}(32)$ & $1.406(2)$ \\
\hline $\mathrm{C}(31)-\mathrm{C}(36)$ & $1.408(2)$ \\
\hline $\mathrm{C}(31)-\mathrm{Li}$ & $2.776(3)$ \\
\hline $\mathrm{C}(32)-\mathrm{C}(33)$ & $1.395(2)$ \\
\hline $\mathrm{C}(32)-\mathrm{C}(37)$ & $1.520(2)$ \\
\hline $\mathrm{C}(33)-\mathrm{C}(34)$ & $1.374(3)$ \\
\hline $\mathrm{C}(34)-\mathrm{C}(35)$ & $1.382(3)$ \\
\hline$C(35)-C(36)$ & $1.387(2)$ \\
\hline$C(36)-C(40)$ & $1.522(2)$ \\
\hline
\end{tabular}




\begin{tabular}{|c|c|}
\hline$C(37)-C(38)$ & $1.521(3)$ \\
\hline $\mathrm{C}(37)-\mathrm{C}(39)$ & $1.525(3)$ \\
\hline $\mathrm{C}(40)-\mathrm{C}(41)$ & $1.519(3)$ \\
\hline$C(40)-C(42)$ & $1.532(3)$ \\
\hline $\mathrm{C}(51)-\mathrm{C}(52)$ & $1.406(3)$ \\
\hline$C(51)-C(56)$ & $1.412(3)$ \\
\hline $\mathrm{C}(51)-\mathrm{Li}$ & $2.784(3)$ \\
\hline$C(52)-C(53)$ & $1.404(2)$ \\
\hline$C(52)-C(57)$ & $1.517(3)$ \\
\hline $\mathrm{C}(53)-\mathrm{C}(54)$ & $1.378(3)$ \\
\hline$C(54)-C(55)$ & $1.369(4)$ \\
\hline$C(55)-C(56)$ & $1.393(3)$ \\
\hline$C(56)-C(60)$ & $1.521(3)$ \\
\hline $\mathrm{C}(57)-\mathrm{C}(58)$ & $1.518(3)$ \\
\hline $\mathrm{C}(57)-\mathrm{C}(59)$ & $1.525(3)$ \\
\hline$C(60)-C(61 A)$ & $1.426(5)$ \\
\hline $\mathrm{C}(60)-\mathrm{C}(62 \mathrm{~B})$ & $1.474(8)$ \\
\hline$C(60)-C(61 B)$ & $1.578(7)$ \\
\hline $\mathrm{C}(60)-\mathrm{C}(62 \mathrm{~A})$ & $1.583(6)$ \\
\hline $\mathrm{Li}-\mathrm{C}(14 \mathrm{~S})$ & $2.674(4)$ \\
\hline Li-C(13S) & $2.732(4)$ \\
\hline$C(10 S)-C(11 S)$ & $1.500(3)$ \\
\hline$C(11 S)-C(12 S)$ & $1.359(4)$ \\
\hline$C(11 S)-C(16 S)$ & $1.377(4)$ \\
\hline $\mathrm{C}(12 \mathrm{~S})-\mathrm{C}(13 \mathrm{~S})$ & $1.372(4)$ \\
\hline$C(13 S)-C(14 S)$ & $1.359(4)$ \\
\hline $\mathrm{C}(14 \mathrm{~S})-\mathrm{C}(15 \mathrm{~S})$ & $1.342(4)$ \\
\hline$C(15 S)-C(16 S)$ & $1.380(4)$ \\
\hline C(3)-P-C(11) & 109.94(7) \\
\hline C(3)-P-C(21) & $103.90(7)$ \\
\hline $\mathrm{C}(11)-\mathrm{P}-\mathrm{C}(21)$ & $105.20(7)$ \\
\hline $\mathrm{C}(2)-\mathrm{N}(1)-\mathrm{C}(31)$ & $121.10(12)$ \\
\hline $\mathrm{C}(2)-\mathrm{N}(1)-\mathrm{Li}$ & $126.66(13)$ \\
\hline $\mathrm{C}(31)-\mathrm{N}(1)-\mathrm{Li}$ & $112.14(12)$ \\
\hline $\mathrm{C}(4)-\mathrm{N}(2)-\mathrm{C}(51)$ & $121.77(13)$ \\
\hline
\end{tabular}




\begin{tabular}{|c|c|}
\hline $\mathrm{C}(4)-\mathrm{N}(2)-\mathrm{Li}$ & $125.95(13)$ \\
\hline $\mathrm{C}(51)-\mathrm{N}(2)-\mathrm{Li}$ & $112.28(13)$ \\
\hline $\mathrm{N}(1)-\mathrm{C}(2)-\mathrm{C}(3)$ & $122.71(13)$ \\
\hline $\mathrm{N}(1)-\mathrm{C}(2)-\mathrm{C}(1)$ & $118.69(12)$ \\
\hline $\mathrm{C}(3)-\mathrm{C}(2)-\mathrm{C}(1)$ & $118.57(12)$ \\
\hline $\mathrm{C}(2)-\mathrm{C}(3)-\mathrm{C}(4)$ & $124.09(13)$ \\
\hline $\mathrm{C}(2)-\mathrm{C}(3)-\mathrm{P}$ & $122.01(10)$ \\
\hline $\mathrm{C}(4)-\mathrm{C}(3)-\mathrm{P}$ & $112.85(10)$ \\
\hline $\mathrm{N}(2)-\mathrm{C}(4)-\mathrm{C}(3)$ & $123.69(13)$ \\
\hline $\mathrm{N}(2)-\mathrm{C}(4)-\mathrm{C}(5)$ & $118.66(14)$ \\
\hline$C(3)-C(4)-C(5)$ & $117.60(14)$ \\
\hline $\mathrm{C}(12)-\mathrm{C}(11)-\mathrm{C}(16)$ & $117.73(15)$ \\
\hline$C(12)-C(11)-P$ & $122.77(12)$ \\
\hline$C(16)-C(11)-P$ & $119.02(13)$ \\
\hline $\mathrm{C}(13)-\mathrm{C}(12)-\mathrm{C}(11)$ & $120.93(16)$ \\
\hline $\mathrm{C}(14)-\mathrm{C}(13)-\mathrm{C}(12)$ & $120.49(18)$ \\
\hline $\mathrm{C}(15)-\mathrm{C}(14)-\mathrm{C}(13)$ & $119.42(18)$ \\
\hline $\mathrm{C}(14)-\mathrm{C}(15)-\mathrm{C}(16)$ & $120.61(18)$ \\
\hline $\mathrm{C}(15)-\mathrm{C}(16)-\mathrm{C}(11)$ & $120.75(17)$ \\
\hline $\mathrm{C}(26)-\mathrm{C}(21)-\mathrm{C}(22)$ & $117.83(15)$ \\
\hline$C(26)-C(21)-P$ & $125.96(12)$ \\
\hline $\mathrm{C}(22)-\mathrm{C}(21)-\mathrm{P}$ & $116.10(12)$ \\
\hline $\mathrm{C}(23)-\mathrm{C}(22)-\mathrm{C}(21)$ & $121.11(16)$ \\
\hline $\mathrm{C}(24)-\mathrm{C}(23)-\mathrm{C}(22)$ & $120.15(18)$ \\
\hline$C(25)-C(24)-C(23)$ & 119.64(17) \\
\hline $\mathrm{C}(24)-\mathrm{C}(25)-\mathrm{C}(26)$ & $120.23(17)$ \\
\hline$C(25)-C(26)-C(21)$ & $121.02(16)$ \\
\hline $\mathrm{C}(32)-\mathrm{C}(31)-\mathrm{C}(36)$ & $120.60(14)$ \\
\hline $\mathrm{C}(32)-\mathrm{C}(31)-\mathrm{N}(1)$ & $120.18(13)$ \\
\hline $\mathrm{C}(36)-\mathrm{C}(31)-\mathrm{N}(1)$ & $118.80(13)$ \\
\hline $\mathrm{C}(32)-\mathrm{C}(31)-\mathrm{Li}$ & $116.25(12)$ \\
\hline $\mathrm{C}(36)-\mathrm{C}(31)-\mathrm{Li}$ & $104.33(12)$ \\
\hline $\mathrm{N}(1)-\mathrm{C}(31)-\mathrm{Li}$ & $39.32(8)$ \\
\hline $\mathrm{C}(33)-\mathrm{C}(32)-\mathrm{C}(31)$ & $118.15(15)$ \\
\hline $\mathrm{C}(33)-\mathrm{C}(32)-\mathrm{C}(37)$ & $120.30(15)$ \\
\hline $\mathrm{C}(31)-\mathrm{C}(32)-\mathrm{C}(37)$ & $121.54(13)$ \\
\hline
\end{tabular}




\begin{tabular}{|c|c|}
\hline $\mathrm{C}(34)-\mathrm{C}(33)-\mathrm{C}(32)$ & $121.57(17)$ \\
\hline $\mathrm{C}(33)-\mathrm{C}(34)-\mathrm{C}(35)$ & $119.80(15)$ \\
\hline $\mathrm{C}(34)-\mathrm{C}(35)-\mathrm{C}(36)$ & $121.07(16)$ \\
\hline $\mathrm{C}(35)-\mathrm{C}(36)-\mathrm{C}(31)$ & 118.81(16) \\
\hline$C(35)-C(36)-C(40)$ & $121.00(15)$ \\
\hline $\mathrm{C}(31)-\mathrm{C}(36)-\mathrm{C}(40)$ & $120.18(14)$ \\
\hline $\mathrm{C}(32)-\mathrm{C}(37)-\mathrm{C}(38)$ & $113.32(15)$ \\
\hline $\mathrm{C}(32)-\mathrm{C}(37)-\mathrm{C}(39)$ & $110.87(15)$ \\
\hline $\mathrm{C}(38)-\mathrm{C}(37)-\mathrm{C}(39)$ & 110.11(17) \\
\hline $\mathrm{C}(41)-\mathrm{C}(40)-\mathrm{C}(36)$ & $111.80(16)$ \\
\hline $\mathrm{C}(41)-\mathrm{C}(40)-\mathrm{C}(42)$ & $109.85(18)$ \\
\hline$C(36)-C(40)-C(42)$ & $113.68(17)$ \\
\hline $\mathrm{C}(52)-\mathrm{C}(51)-\mathrm{C}(56)$ & 121.41(16) \\
\hline $\mathrm{C}(52)-\mathrm{C}(51)-\mathrm{N}(2)$ & $120.35(16)$ \\
\hline $\mathrm{C}(56)-\mathrm{C}(51)-\mathrm{N}(2)$ & $117.84(16)$ \\
\hline $\mathrm{C}(52)-\mathrm{C}(51)-\mathrm{Li}$ & $113.88(13)$ \\
\hline $\mathrm{C}(56)-\mathrm{C}(51)-\mathrm{Li}$ & $106.07(13)$ \\
\hline $\mathrm{N}(2)-\mathrm{C}(51)-\mathrm{Li}$ & $39.35(9)$ \\
\hline $\mathrm{C}(53)-\mathrm{C}(52)-\mathrm{C}(51)$ & 117.13(19) \\
\hline$C(53)-C(52)-C(57)$ & $121.16(18)$ \\
\hline $\mathrm{C}(51)-\mathrm{C}(52)-\mathrm{C}(57)$ & $121.70(15)$ \\
\hline $\mathrm{C}(54)-\mathrm{C}(53)-\mathrm{C}(52)$ & $121.6(2)$ \\
\hline $\mathrm{C}(55)-\mathrm{C}(54)-\mathrm{C}(53)$ & $120.50(19)$ \\
\hline $\mathrm{C}(54)-\mathrm{C}(55)-\mathrm{C}(56)$ & $120.9(2)$ \\
\hline $\mathrm{C}(55)-\mathrm{C}(56)-\mathrm{C}(51)$ & $118.4(2)$ \\
\hline $\mathrm{C}(55)-\mathrm{C}(56)-\mathrm{C}(60)$ & $120.4(2)$ \\
\hline $\mathrm{C}(51)-\mathrm{C}(56)-\mathrm{C}(60)$ & $121.24(17)$ \\
\hline $\mathrm{C}(52)-\mathrm{C}(57)-\mathrm{C}(58)$ & $111.44(17)$ \\
\hline $\mathrm{C}(52)-\mathrm{C}(57)-\mathrm{C}(59)$ & $112.86(16)$ \\
\hline $\mathrm{C}(58)-\mathrm{C}(57)-\mathrm{C}(59)$ & 108.81(17) \\
\hline$C(61 A)-C(60)-C(62 B)$ & $125.5(4)$ \\
\hline$C(61 A)-C(60)-C(56)$ & $112.0(3)$ \\
\hline$C(62 B)-C(60)-C(56)$ & $121.2(4)$ \\
\hline$C(61 A)-C(60)-C(61 B)$ & $35.4(3)$ \\
\hline $\mathrm{C}(62 \mathrm{~B})-\mathrm{C}(60)-\mathrm{C}(61 \mathrm{~B})$ & $107.5(4)$ \\
\hline$C(56)-C(60)-C(61 B)$ & $112.8(3)$ \\
\hline
\end{tabular}




$\begin{array}{lc}\text { C(61A)-C(60)-C(62A) } & 111.9(4) \\ \text { C(62B)-C(60)-C(62A) } & 40.7(3) \\ \text { C(56)-C(60)-C(62A) } & 109.1(3) \\ \text { C(61B)-C(60)-C(62A) } & 79.3(4) \\ \text { N(1)-Li-N(2) } & 94.99(13) \\ \text { N(1)-Li-C(14S) } & 150.22(16) \\ \text { N(2)-Li-C(14S) } & 108.35(13) \\ \text { N(1)-Li-C(13S) } & 121.15(15) \\ \text { N(2)-Li-C(13S) } & 129.68(15) \\ \text { C(14S)-Li-C(13S) } & 29.08(9) \\ \text { N(1)-Li-C(31) } & 28.54(6) \\ \text { N(2)-Li-C(31) } & 123.20(13) \\ \text { C(14S)-Li-C(31) } & 123.79(12) \\ \text { C(13S)-Li-C(31) } & 95.44(12) \\ \text { N(1)-Li-C(51) } & 123.02(14) \\ \text { N(2)-Li-C(51) } & 28.37(6) \\ \text { C(14S)-Li-C(51) } & 80.41(10) \\ \text { C(13S)-Li-C(51) } & 104.83(12) \\ \text { C(31)-Li-C(51) } & 150.35(12) \\ \text { C(12S)-C(11S)-C(16S) } & 116.9(2) \\ \text { C(12S)-C(11S)-C(10S) } & 121.8(3) \\ \text { C(16S)-C(11S)-C(10S) } & 121.3(3) \\ \text { C(11S)-C(12S)-C(13S) } & 121.3(2) \\ \text { C(14S)-C(13S)-C(12S) } & 121.0(3) \\ \text { C(14S)-C(13S)-Li } & 73.09(17) \\ \text { C(12S)-C(13S)-Li } & 93.6(2) \\ \text { C(15S)-C(14S)-C(13S) } & 118.9(2) \\ \text { C(15S)-C(14S)-Li } & 89.52(19) \\ \text { C(13S)-C(14S)-Li } & 77.82(18) \\ \text { C(14S)-C(15S)-C(16S) } & 120.4(2) \\ \text { C(11S)-C(16S)-C(15S) } & 121.5(3) \\ & \end{array}$

Symmetry transformations used to generate equivalent atoms: 
Table S9. Anisotropic displacement parameters $\left(\AA^{2} \times 10^{3}\right)$ for $5 \mathbf{L i}$. The anisotropic displacement factor exponent takes the form: $-2 \pi^{2}\left[h^{2} a^{* 2} U^{11}+\ldots+2 h k a^{*} b^{*} U^{12}\right]$

\begin{tabular}{|c|c|c|c|c|c|c|}
\hline & $\mathrm{U}^{11}$ & $\mathrm{U}^{22}$ & $\mathrm{U}^{33}$ & $\mathrm{U}^{23}$ & $\mathrm{U}^{13}$ & $\mathrm{U}^{12}$ \\
\hline $\mathrm{P}$ & $37(1)$ & 21(1) & $27(1)$ & $-5(1)$ & $1(1)$ & $-6(1)$ \\
\hline $\mathrm{N}(1)$ & $35(1)$ & 21(1) & $25(1)$ & $-3(1)$ & $-3(1)$ & $-5(1)$ \\
\hline $\mathrm{N}(2)$ & $44(1)$ & $27(1)$ & $24(1)$ & $-3(1)$ & $3(1)$ & $-7(1)$ \\
\hline $\mathrm{C}(1)$ & $35(1)$ & $23(1)$ & $27(1)$ & $-3(1)$ & $1(1)$ & $-5(1)$ \\
\hline $\mathrm{C}(2)$ & $29(1)$ & $24(1)$ & $23(1)$ & $-5(1)$ & $-4(1)$ & $-7(1)$ \\
\hline $\mathrm{C}(3)$ & $33(1)$ & 21(1) & $25(1)$ & $-5(1)$ & $0(1)$ & $-5(1)$ \\
\hline $\mathrm{C}(4)$ & $41(1)$ & $23(1)$ & $28(1)$ & $-3(1)$ & $-1(1)$ & $-6(1)$ \\
\hline $\mathrm{C}(5)$ & $88(2)$ & $27(1)$ & $33(1)$ & $0(1)$ & $11(1)$ & $1(1)$ \\
\hline $\mathrm{C}(11)$ & $38(1)$ & $24(1)$ & $29(1)$ & $-1(1)$ & $-2(1)$ & $1(1)$ \\
\hline $\mathrm{C}(12)$ & $39(1)$ & $34(1)$ & $35(1)$ & $-7(1)$ & $-6(1)$ & $-1(1)$ \\
\hline $\mathrm{C}(13)$ & $37(1)$ & $47(1)$ & $53(1)$ & $-2(1)$ & $-10(1)$ & $-4(1)$ \\
\hline $\mathrm{C}(14)$ & $35(1)$ & $49(1)$ & $56(1)$ & $-1(1)$ & $2(1)$ & $9(1)$ \\
\hline$C(15)$ & $52(1)$ & $38(1)$ & $49(1)$ & $-9(1)$ & $5(1)$ & 11(1) \\
\hline$C(16)$ & $45(1)$ & $32(1)$ & $39(1)$ & $-9(1)$ & $-1(1)$ & $2(1)$ \\
\hline $\mathrm{C}(21)$ & $35(1)$ & $24(1)$ & $33(1)$ & $-8(1)$ & $-1(1)$ & $-10(1)$ \\
\hline $\mathrm{C}(22)$ & $37(1)$ & $42(1)$ & $42(1)$ & $-11(1)$ & $1(1)$ & $-13(1)$ \\
\hline $\mathrm{C}(23)$ & $35(1)$ & $56(1)$ & $59(1)$ & $-12(1)$ & $-10(1)$ & $-11(1)$ \\
\hline$C(24)$ & $54(1)$ & $55(1)$ & $49(1)$ & $-11(1)$ & $-22(1)$ & $-8(1)$ \\
\hline$C(25)$ & $59(1)$ & $52(1)$ & $33(1)$ & $-10(1)$ & $-7(1)$ & $-7(1)$ \\
\hline$C(26)$ & $39(1)$ & $40(1)$ & $34(1)$ & $-10(1)$ & $-1(1)$ & $-6(1)$ \\
\hline $\mathrm{C}(31)$ & $33(1)$ & $22(1)$ & $27(1)$ & $-5(1)$ & $5(1)$ & $-5(1)$ \\
\hline $\mathrm{C}(32)$ & $31(1)$ & $25(1)$ & $34(1)$ & $-4(1)$ & $2(1)$ & $-2(1)$ \\
\hline $\mathrm{C}(33)$ & $38(1)$ & $28(1)$ & $49(1)$ & 1(1) & $-3(1)$ & $2(1)$ \\
\hline $\mathrm{C}(34)$ & $48(1)$ & $20(1)$ & $64(1)$ & $-4(1)$ & $6(1)$ & $-3(1)$ \\
\hline $\mathrm{C}(35)$ & $48(1)$ & $28(1)$ & $54(1)$ & $-14(1)$ & $7(1)$ & $-14(1)$ \\
\hline$C(36)$ & $42(1)$ & $29(1)$ & $34(1)$ & $-10(1)$ & $3(1)$ & $-9(1)$ \\
\hline $\mathrm{C}(37)$ & $37(1)$ & $33(1)$ & $39(1)$ & $-4(1)$ & $-9(1)$ & $-1(1)$ \\
\hline $\mathrm{C}(38)$ & $88(2)$ & $59(1)$ & $46(1)$ & $-6(1)$ & $-21(1)$ & $-16(1)$ \\
\hline $\mathrm{C}(39)$ & $45(1)$ & $88(2)$ & $76(2)$ & $-30(1)$ & $0(1)$ & $-24(1)$ \\
\hline $\mathrm{C}(40)$ & $64(1)$ & $40(1)$ & $45(1)$ & $-12(1)$ & $-15(1)$ & $-17(1)$ \\
\hline $\mathrm{C}(41)$ & $64(1)$ & $80(2)$ & $88(2)$ & $-45(1)$ & $-39(1)$ & $14(1)$ \\
\hline
\end{tabular}




\begin{tabular}{|c|c|c|c|c|c|c|}
\hline $\mathrm{C}(42)$ & $77(2)$ & $71(2)$ & $58(1)$ & $-31(1)$ & $-11(1)$ & $-25(1)$ \\
\hline $\mathrm{C}(51)$ & $55(1)$ & $25(1)$ & $29(1)$ & $-3(1)$ & $8(1)$ & $-5(1)$ \\
\hline $\mathrm{C}(52)$ & $68(1)$ & $27(1)$ & $28(1)$ & $-6(1)$ & $3(1)$ & $-5(1)$ \\
\hline $\mathrm{C}(53)$ & $98(2)$ & $41(1)$ & $28(1)$ & $-8(1)$ & $6(1)$ & $-10(1)$ \\
\hline $\mathrm{C}(54)$ & $98(2)$ & $56(1)$ & $43(1)$ & $-9(1)$ & $35(1)$ & $-18(1)$ \\
\hline $\mathrm{C}(55)$ & $68(1)$ & $57(1)$ & $57(1)$ & $-3(1)$ & $26(1)$ & $-14(1)$ \\
\hline$C(56)$ & $56(1)$ & $37(1)$ & $49(1)$ & $-1(1)$ & $14(1)$ & $-9(1)$ \\
\hline $\mathrm{C}(57)$ & $67(1)$ & $40(1)$ & $31(1)$ & $-6(1)$ & $-6(1)$ & $-9(1)$ \\
\hline $\mathrm{C}(58)$ & $78(2)$ & $39(1)$ & $81(2)$ & $1(1)$ & $-22(1)$ & $-12(1)$ \\
\hline C(59) & $84(2)$ & $42(1)$ & $69(1)$ & $0(1)$ & $-21(1)$ & $-2(1)$ \\
\hline $\mathrm{C}(60)$ & $55(1)$ & $69(2)$ & $64(1)$ & $8(1)$ & $-2(1)$ & $-24(1)$ \\
\hline $\mathrm{Li}$ & $54(2)$ & $31(1)$ & $32(1)$ & $-4(1)$ & $7(1)$ & $-1(1)$ \\
\hline $\mathrm{C}(10 \mathrm{~S})$ & $163(3)$ & $72(2)$ & $57(2)$ & $-4(1)$ & $-19(2)$ & $6(2)$ \\
\hline $\mathrm{C}(11 \mathrm{~S})$ & $86(2)$ & $48(1)$ & $41(1)$ & $-13(1)$ & $5(1)$ & $-2(1)$ \\
\hline $\mathrm{C}(12 \mathrm{~S})$ & $66(2)$ & $54(1)$ & $102(2)$ & $-10(1)$ & $25(2)$ & $-13(1)$ \\
\hline $\mathrm{C}(13 \mathrm{~S})$ & $58(2)$ & $67(2)$ & $119(2)$ & $-19(2)$ & $-7(2)$ & $-1(1)$ \\
\hline$C(14 S)$ & $76(2)$ & $54(1)$ & $59(1)$ & $-12(1)$ & $1(1)$ & $12(1)$ \\
\hline$C(15 S)$ & $86(2)$ & $53(1)$ & $86(2)$ & $2(1)$ & $8(2)$ & $-19(1)$ \\
\hline$C(16 S)$ & $84(2)$ & $66(2)$ & $99(2)$ & $-8(2)$ & $-27(2)$ & $-24(1)$ \\
\hline
\end{tabular}




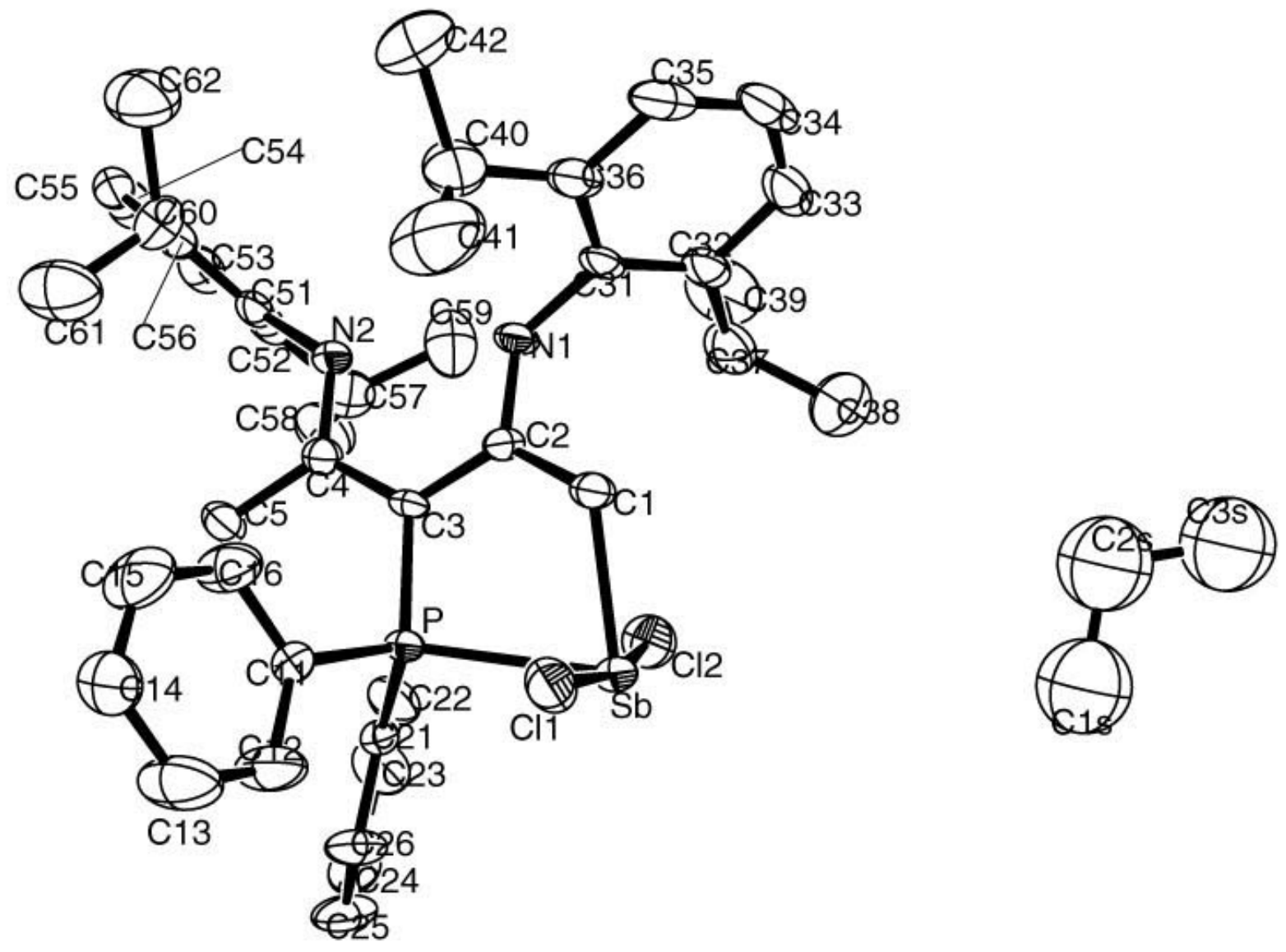

Figure S3: Solid State Structure of $\mathbf{6 S b}$. Ellipsoids are 50\% probability, hydrogen atoms are removed for clarity. This highlights the complete numbering scheme and includes the $n$-pentane solvate. 
Table S10. Crystal data and structure refinement for $\mathbf{6 S b}$.

Identification code

Empirical formula

Formula weight

Temperature

Wavelength

Crystal system

Space group

Unit cell dimensions

Volume

Z

Density (calculated)

Absorption coefficient

$\mathrm{F}(000)$

Crystal size

Theta range for data collection

Index ranges

Reflections collected

Independent reflections

Completeness to theta $=26.38^{\circ}$

Absorption correction

Max. and min. transmission

Refinement method

Data / restraints / parameters

Goodness-of-fit on $\mathrm{F}^{2}$

Final $\mathrm{R}$ indices [I $>2 \operatorname{sigma}(\mathrm{I})]$

$\mathrm{R}$ indices (all data)

Largest diff. peak and hole dal0343

C43.50 H56 Cl2 N2 P Sb

830.52

193(2) K

$0.71073 \AA$

Monoclinic

$\mathrm{C} 2 / \mathrm{c}$

$\mathrm{a}=34.699(3) \AA \quad \alpha=90^{\circ}$.

$\mathrm{b}=11.3045(9) \AA \quad \beta=96.0247(15)^{\circ}$.

$\mathrm{c}=20.9953(15) \AA \quad \gamma=90^{\circ}$.

8190.0(11) $\AA^{3}$

8

$1.347 \mathrm{Mg} / \mathrm{m}^{3}$

$0.875 \mathrm{~mm}^{-1}$

3448

$0.78 \times 0.14 \times 0.11 \mathrm{~mm}^{3}$

1.90 to $26.38^{\circ}$.

$-43<=\mathrm{h}<=42,-14<=\mathrm{k}<=11,-26<=\mathrm{k}<=25$

21380

$8350[\mathrm{R}($ int $)=0.0606]$

$99.6 \%$

Empirical

0.9099 and 0.5484

Full-matrix least-squares on $\mathrm{F}^{2}$

8350 / 6 / 435

1.027

$\mathrm{R} 1=0.0553, \mathrm{wR} 2=0.1386$

$\mathrm{R} 1=0.0882, \mathrm{wR} 2=0.1525$

1.856 and -1.251 e. $\AA^{-3}$ 
Table S11. Atomic coordinates ( x 10 $)$ and equivalent isotropic displacement parameters $\left(\AA^{2} \times 10^{3}\right)$ for $\mathbf{6 S b}$. $U(\mathrm{eq})$ is defined as one third of the trace of the orthogonalized $\mathrm{U}^{\mathrm{ij}}$ tensor.

\begin{tabular}{|c|c|c|c|c|}
\hline & $\mathrm{x}$ & $\mathrm{y}$ & $\mathrm{z}$ & $\mathrm{U}(\mathrm{eq})$ \\
\hline $\mathrm{Sb}$ & $576(1)$ & $405(1)$ & $326(1)$ & $30(1)$ \\
\hline $\mathrm{Cl}(1)$ & 137(1) & $-1554(1)$ & $332(1)$ & $41(1)$ \\
\hline $\mathrm{Cl}(2)$ & 1052(1) & $2057(1)$ & $214(1)$ & $47(1)$ \\
\hline $\mathrm{P}$ & 1180(1) & $-969(1)$ & $458(1)$ & $22(1)$ \\
\hline $\mathrm{N}(1)$ & $1142(1)$ & $-183(4)$ & $-1383(2)$ & $26(1)$ \\
\hline $\mathrm{N}(2)$ & $1824(1)$ & $-1047(4)$ & $-1027(2)$ & $26(1)$ \\
\hline $\mathrm{C}(1)$ & $649(1)$ & $-87(5)$ & $-655(2)$ & $30(1)$ \\
\hline $\mathrm{C}(2)$ & $1057(1)$ & $-350(4)$ & $-782(2)$ & $23(1)$ \\
\hline$C(3)$ & 1341(1) & $-818(4)$ & $-317(2)$ & $23(1)$ \\
\hline $\mathrm{C}(4)$ & $1720(1)$ & $-1206(4)$ & $-458(2)$ & $24(1)$ \\
\hline$C(5)$ & $1995(2)$ & $-1778(5)$ & $56(2)$ & $36(1)$ \\
\hline $\mathrm{C}(11)$ & 1108(1) & $-2530(4)$ & $615(2)$ & $26(1)$ \\
\hline $\mathrm{C}(12)$ & $969(2)$ & $-2910(6)$ & 1167(3) & $60(2)$ \\
\hline $\mathrm{C}(13)$ & $912(3)$ & $-4098(7)$ & 1277(3) & $77(3)$ \\
\hline $\mathrm{C}(14)$ & $995(2)$ & $-4925(6)$ & $819(3)$ & $56(2)$ \\
\hline $\mathrm{C}(15)$ & $1110(2)$ & $-4539(6)$ & 273(3) & $64(2)$ \\
\hline$C(16)$ & $1174(2)$ & $-3355(6)$ & $161(3)$ & $48(2)$ \\
\hline $\mathrm{C}(21)$ & $1520(1)$ & $-322(4)$ & 1081(2) & $25(1)$ \\
\hline $\mathrm{C}(22)$ & $1802(2)$ & $455(5)$ & $928(3)$ & $40(1)$ \\
\hline $\mathrm{C}(23)$ & $2047(2)$ & $988(6)$ & 1402(3) & $48(2)$ \\
\hline$C(24)$ & $2016(2)$ & $761(6)$ & 2026(3) & $47(2)$ \\
\hline $\mathrm{C}(25)$ & $1727(2)$ & $41(7)$ & 2196(3) & $50(2)$ \\
\hline$C(26)$ & $1480(2)$ & $-505(6)$ & 1721(2) & $41(1)$ \\
\hline $\mathrm{C}(31)$ & $874(1)$ & $150(5)$ & $-1917(2)$ & $27(1)$ \\
\hline $\mathrm{C}(32)$ & $776(2)$ & $1342(5)$ & $-2015(2)$ & $34(1)$ \\
\hline$C(33)$ & $514(2)$ & $1610(6)$ & $-2547(3)$ & $46(2)$ \\
\hline $\mathrm{C}(34)$ & $362(2)$ & $745(7)$ & $-2962(3)$ & $47(2)$ \\
\hline$C(35)$ & $467(2)$ & $-403(7)$ & $-2863(2)$ & $46(2)$ \\
\hline$C(36)$ & $727(2)$ & $-746(6)$ & $-2342(2)$ & $39(1)$ \\
\hline$C(37)$ & $950(2)$ & $2324(5)$ & $-1587(3)$ & $47(2)$ \\
\hline $\mathrm{C}(38)$ & $643(3)$ & $3195(7)$ & $-1401(4)$ & $78(2)$ \\
\hline
\end{tabular}




\begin{tabular}{lrrrr}
$\mathrm{C}(39)$ & $1268(2)$ & $2954(7)$ & $-1910(4)$ & $74(2)$ \\
$\mathrm{C}(40)$ & $853(2)$ & $-2020(6)$ & $-2251(3)$ & $61(2)$ \\
$\mathrm{C}(41)$ & $597(4)$ & $-2693(8)$ & $-1855(5)$ & $121(4)$ \\
$\mathrm{C}(42)$ & $893(3)$ & $-2660(8)$ & $-2875(4)$ & $88(3)$ \\
$\mathrm{C}(51)$ & $2197(1)$ & $-1377(5)$ & $-1202(2)$ & $28(1)$ \\
$\mathrm{C}(52)$ & $2500(2)$ & $-542(5)$ & $-1112(2)$ & $35(1)$ \\
$\mathrm{C}(53)$ & $2850(2)$ & $-828(6)$ & $-1347(3)$ & $42(2)$ \\
$\mathrm{C}(54)$ & $2896(2)$ & $-1867(6)$ & $-1656(3)$ & $48(2)$ \\
$\mathrm{C}(55)$ & $2601(2)$ & $-2671(6)$ & $-1732(3)$ & $44(2)$ \\
$\mathrm{C}(56)$ & $2242(2)$ & $-2452(5)$ & $-1515(2)$ & $34(1)$ \\
$\mathrm{C}(57)$ & $2447(2)$ & $628(6)$ & $-778(3)$ & $43(1)$ \\
$\mathrm{C}(58)$ & $2822(2)$ & $1204(7)$ & $-486(3)$ & $60(2)$ \\
$\mathrm{C}(59)$ & $2227(2)$ & $1489(6)$ & $-1224(4)$ & $58(2)$ \\
$\mathrm{C}(60)$ & $1919(2)$ & $-3353(5)$ & $-1598(3)$ & $46(2)$ \\
$\mathrm{C}(61)$ & $1972(3)$ & $-4329(7)$ & $-1103(4)$ & $76(2)$ \\
$\mathrm{C}(62)$ & $1868(3)$ & $-3891(7)$ & $-2273(3)$ & $77(2)$ \\
$\mathrm{C}(1 S)$ & $36(4)$ & $5650(2)$ & $-87(2)$ & $142(3)$ \\
$\mathrm{C}(2 S)$ & $55(4)$ & $5762(9)$ & $-814(3)$ & $142(3)$ \\
$\mathrm{C}(3 \mathrm{~S})$ & $-199(6)$ & $6805(17)$ & $-1079(7)$ & $142(3)$ \\
\hline & & & & \\
\hline & & & &
\end{tabular}


Table S12. Bond lengths $[\AA ̊]$ and angles $\left[{ }^{\circ}\right]$ for $\mathbf{6 S b}$.

\begin{tabular}{|c|c|}
\hline $\mathrm{Sb}-\mathrm{C}(1)$ & $2.174(5)$ \\
\hline $\mathrm{Sb}-\mathrm{Cl}(2)$ & $2.5212(16)$ \\
\hline Sb-P & $2.6011(13)$ \\
\hline $\mathrm{Sb}-\mathrm{Cl}(1)$ & $2.6885(16)$ \\
\hline $\mathrm{P}-\mathrm{C}(3)$ & $1.783(4)$ \\
\hline P-C(11) & $1.817(5)$ \\
\hline P-C (21) & $1.821(5)$ \\
\hline $\mathrm{N}(1)-\mathrm{C}(2)$ & $1.339(5)$ \\
\hline $\mathrm{N}(1)-\mathrm{C}(31)$ & $1.432(6)$ \\
\hline $\mathrm{N}(2)-\mathrm{C}(4)$ & $1.297(6)$ \\
\hline $\mathrm{N}(2)-\mathrm{C}(51)$ & $1.431(6)$ \\
\hline $\mathrm{C}(1)-\mathrm{C}(2)$ & $1.496(6)$ \\
\hline $\mathrm{C}(2)-\mathrm{C}(3)$ & $1.414(6)$ \\
\hline $\mathrm{C}(3)-\mathrm{C}(4)$ & $1.446(6)$ \\
\hline $\mathrm{C}(4)-\mathrm{C}(5)$ & $1.510(7)$ \\
\hline$C(11)-C(16)$ & $1.370(7)$ \\
\hline $\mathrm{C}(11)-\mathrm{C}(12)$ & $1.372(7)$ \\
\hline $\mathrm{C}(12)-\mathrm{C}(13)$ & $1.380(9)$ \\
\hline $\mathrm{C}(13)-\mathrm{C}(14)$ & $1.394(10)$ \\
\hline$C(14)-C(15)$ & $1.325(9)$ \\
\hline$C(15)-C(16)$ & $1.382(9)$ \\
\hline$C(21)-C(22)$ & $1.378(7)$ \\
\hline$C(21)-C(26)$ & $1.379(7)$ \\
\hline$C(22)-C(23)$ & $1.379(8)$ \\
\hline$C(23)-C(24)$ & $1.350(8)$ \\
\hline$C(24)-C(25)$ & $1.370(9)$ \\
\hline$C(25)-C(26)$ & $1.389(8)$ \\
\hline $\mathrm{C}(31)-\mathrm{C}(32)$ & $1.400(8)$ \\
\hline$C(31)-C(36)$ & $1.409(8)$ \\
\hline $\mathrm{C}(32)-\mathrm{C}(33)$ & $1.398(8)$ \\
\hline $\mathrm{C}(32)-\mathrm{C}(37)$ & $1.513(8)$ \\
\hline $\mathrm{C}(33)-\mathrm{C}(34)$ & $1.378(9)$ \\
\hline$C(34)-C(35)$ & $1.357(9)$ \\
\hline$C(35)-C(36)$ & $1.398(8)$ \\
\hline
\end{tabular}




\begin{tabular}{|c|c|}
\hline $\mathrm{C}(36)-\mathrm{C}(40)$ & $1.512(9)$ \\
\hline $\mathrm{C}(37)-\mathrm{C}(39)$ & $1.530(9)$ \\
\hline $\mathrm{C}(37)-\mathrm{C}(38)$ & $1.533(9)$ \\
\hline $\mathrm{C}(40)-\mathrm{C}(41)$ & $1.487(12)$ \\
\hline$C(40)-C(42)$ & $1.517(9)$ \\
\hline$C(51)-C(56)$ & $1.399(7)$ \\
\hline$C(51)-C(52)$ & $1.411(8)$ \\
\hline$C(52)-C(53)$ & $1.397(7)$ \\
\hline$C(52)-C(57)$ & $1.516(8)$ \\
\hline $\mathrm{C}(53)-\mathrm{C}(54)$ & $1.360(9)$ \\
\hline$C(54)-C(55)$ & $1.367(9)$ \\
\hline$C(55)-C(56)$ & $1.391(7)$ \\
\hline$C(56)-C(60)$ & $1.512(8)$ \\
\hline $\mathrm{C}(57)-\mathrm{C}(59)$ & $1.503(9)$ \\
\hline $\mathrm{C}(57)-\mathrm{C}(58)$ & $1.523(8)$ \\
\hline $\mathrm{C}(60)-\mathrm{C}(61)$ & $1.514(10)$ \\
\hline$C(60)-C(62)$ & $1.536(9)$ \\
\hline$C(1 S)-C(2 S)$ & $1.5389(10)$ \\
\hline $\mathrm{C}(1 \mathrm{~S})-\mathrm{C}(1 \mathrm{~S}) \# 1$ & $1.5401(10)$ \\
\hline$C(2 S)-C(3 S)$ & $1.5397(10)$ \\
\hline $\mathrm{C}(1)-\mathrm{Sb}-\mathrm{Cl}(2)$ & $87.64(15)$ \\
\hline $\mathrm{C}(1)-\mathrm{Sb}-\mathrm{P}$ & $77.00(13)$ \\
\hline $\mathrm{Cl}(2)-\mathrm{Sb}-\mathrm{P}$ & $85.60(5)$ \\
\hline $\mathrm{C}(1)-\mathrm{Sb}-\mathrm{Cl}(1)$ & $85.23(15)$ \\
\hline $\mathrm{Cl}(2)-\mathrm{Sb}-\mathrm{Cl}(1)$ & 171.11(5) \\
\hline $\mathrm{P}-\mathrm{Sb}-\mathrm{Cl}(1)$ & $87.63(4)$ \\
\hline $\mathrm{C}(3)-\mathrm{P}-\mathrm{C}(11)$ & $108.7(2)$ \\
\hline $\mathrm{C}(3)-\mathrm{P}-\mathrm{C}(21)$ & $112.2(2)$ \\
\hline $\mathrm{C}(11)-\mathrm{P}-\mathrm{C}(21)$ & $110.5(2)$ \\
\hline $\mathrm{C}(3)-\mathrm{P}-\mathrm{Sb}$ & $99.85(16)$ \\
\hline $\mathrm{C}(11)-\mathrm{P}-\mathrm{Sb}$ & $118.30(16)$ \\
\hline $\mathrm{C}(21)-\mathrm{P}-\mathrm{Sb}$ & $106.89(16)$ \\
\hline $\mathrm{C}(2)-\mathrm{N}(1)-\mathrm{C}(31)$ & $125.8(4)$ \\
\hline $\mathrm{C}(4)-\mathrm{N}(2)-\mathrm{C}(51)$ & $123.1(4)$ \\
\hline $\mathrm{C}(2)-\mathrm{C}(1)-\mathrm{Sb}$ & $115.2(3)$ \\
\hline
\end{tabular}




\begin{tabular}{|c|c|}
\hline $\mathrm{N}(1)-\mathrm{C}(2)-\mathrm{C}(3)$ & $119.7(4)$ \\
\hline $\mathrm{N}(1)-\mathrm{C}(2)-\mathrm{C}(1)$ & $116.5(4)$ \\
\hline$C(3)-C(2)-C(1)$ & $123.7(4)$ \\
\hline$C(2)-C(3)-C(4)$ & $123.8(4)$ \\
\hline$C(2)-C(3)-P$ & $113.8(3)$ \\
\hline$C(4)-C(3)-P$ & $122.3(3)$ \\
\hline $\mathrm{N}(2)-\mathrm{C}(4)-\mathrm{C}(3)$ & $119.5(4)$ \\
\hline $\mathrm{N}(2)-\mathrm{C}(4)-\mathrm{C}(5)$ & $120.2(4)$ \\
\hline$C(3)-C(4)-C(5)$ & $120.3(4)$ \\
\hline $\mathrm{C}(16)-\mathrm{C}(11)-\mathrm{C}(12)$ & $118.3(5)$ \\
\hline$C(16)-C(11)-P$ & $119.9(4)$ \\
\hline $\mathrm{C}(12)-\mathrm{C}(11)-\mathrm{P}$ & $121.7(4)$ \\
\hline $\mathrm{C}(11)-\mathrm{C}(12)-\mathrm{C}(13)$ & $121.1(6)$ \\
\hline $\mathrm{C}(12)-\mathrm{C}(13)-\mathrm{C}(14)$ & $119.5(6)$ \\
\hline $\mathrm{C}(15)-\mathrm{C}(14)-\mathrm{C}(13)$ & $118.6(6)$ \\
\hline $\mathrm{C}(14)-\mathrm{C}(15)-\mathrm{C}(16)$ & $122.3(6)$ \\
\hline $\mathrm{C}(11)-\mathrm{C}(16)-\mathrm{C}(15)$ & $119.9(6)$ \\
\hline $\mathrm{C}(22)-\mathrm{C}(21)-\mathrm{C}(26)$ & $118.0(5)$ \\
\hline $\mathrm{C}(22)-\mathrm{C}(21)-\mathrm{P}$ & $120.6(4)$ \\
\hline$C(26)-C(21)-P$ & $121.1(4)$ \\
\hline $\mathrm{C}(21)-\mathrm{C}(22)-\mathrm{C}(23)$ & $120.6(5)$ \\
\hline $\mathrm{C}(24)-\mathrm{C}(23)-\mathrm{C}(22)$ & $120.8(6)$ \\
\hline $\mathrm{C}(23)-\mathrm{C}(24)-\mathrm{C}(25)$ & $120.1(5)$ \\
\hline $\mathrm{C}(24)-\mathrm{C}(25)-\mathrm{C}(26)$ & 119.3(5) \\
\hline$C(21)-C(26)-C(25)$ & $121.1(5)$ \\
\hline $\mathrm{C}(32)-\mathrm{C}(31)-\mathrm{C}(36)$ & $122.2(5)$ \\
\hline $\mathrm{C}(32)-\mathrm{C}(31)-\mathrm{N}(1)$ & $119.7(5)$ \\
\hline $\mathrm{C}(36)-\mathrm{C}(31)-\mathrm{N}(1)$ & $118.1(5)$ \\
\hline $\mathrm{C}(33)-\mathrm{C}(32)-\mathrm{C}(31)$ & $117.0(5)$ \\
\hline $\mathrm{C}(33)-\mathrm{C}(32)-\mathrm{C}(37)$ & $120.1(6)$ \\
\hline $\mathrm{C}(31)-\mathrm{C}(32)-\mathrm{C}(37)$ & $122.9(5)$ \\
\hline $\mathrm{C}(34)-\mathrm{C}(33)-\mathrm{C}(32)$ & $121.7(6)$ \\
\hline$C(35)-C(34)-C(33)$ & $120.2(5)$ \\
\hline$C(34)-C(35)-C(36)$ & $121.7(6)$ \\
\hline$C(35)-C(36)-C(31)$ & $117.2(6)$ \\
\hline$C(35)-C(36)-C(40)$ & $121.2(6)$ \\
\hline
\end{tabular}




$\begin{array}{ll}\mathrm{C}(31)-\mathrm{C}(36)-\mathrm{C}(40) & 121.6(5) \\ \mathrm{C}(32)-\mathrm{C}(37)-\mathrm{C}(39) & 109.9(5) \\ \mathrm{C}(32)-\mathrm{C}(37)-\mathrm{C}(38) & 112.2(6) \\ \mathrm{C}(39)-\mathrm{C}(37)-\mathrm{C}(38) & 111.6(6) \\ \mathrm{C}(41)-\mathrm{C}(40)-\mathrm{C}(36) & 112.1(7) \\ \mathrm{C}(41)-\mathrm{C}(40)-\mathrm{C}(42) & 110.7(7) \\ \mathrm{C}(36)-\mathrm{C}(40)-\mathrm{C}(42) & 113.3(6) \\ \mathrm{C}(56)-\mathrm{C}(51)-\mathrm{C}(52) & 121.7(5) \\ \mathrm{C}(56)-\mathrm{C}(51)-\mathrm{N}(2) & 119.8(5) \\ \mathrm{C}(52)-\mathrm{C}(51)-\mathrm{N}(2) & 118.2(5) \\ \mathrm{C}(53)-\mathrm{C}(52)-\mathrm{C}(51) & 117.5(5) \\ \mathrm{C}(53)-\mathrm{C}(52)-\mathrm{C}(57) & 121.0(5) \\ \mathrm{C}(51)-\mathrm{C}(52)-\mathrm{C}(57) & 121.4(5) \\ \mathrm{C}(54)-\mathrm{C}(53)-\mathrm{C}(52) & 121.2(6) \\ \mathrm{C}(53)-\mathrm{C}(54)-\mathrm{C}(55) & 120.4(5) \\ \mathrm{C}(54)-\mathrm{C}(55)-\mathrm{C}(56) & 122.0(6) \\ \mathrm{C}(55)-\mathrm{C}(56)-\mathrm{C}(51) & 117.2(5) \\ \mathrm{C}(55)-\mathrm{C}(56)-\mathrm{C}(60) & 121.4(5) \\ \mathrm{C}(51)-\mathrm{C}(56)-\mathrm{C}(60) & 121.4(4) \\ \mathrm{C}(59)-\mathrm{C}(57)-\mathrm{C}(52) & 110.8(5) \\ \mathrm{C}(59)-\mathrm{C}(57)-\mathrm{C}(58) & 109.3(5) \\ \mathrm{C}(52)-\mathrm{C}(57)-\mathrm{C}(58) & 114.8(5) \\ \mathrm{C}(56)-\mathrm{C}(60)-\mathrm{C}(61) & 112.1(6) \\ \mathrm{C}(56)-\mathrm{C}(60)-\mathrm{C}(62) & 112.6(5) \\ \mathrm{C}(61)-\mathrm{C}(60)-\mathrm{C}(62) & 109.8(6) \\ \mathrm{C}(2 \mathrm{~S})-\mathrm{C}(1 \mathrm{~S})-\mathrm{C}(1 \mathrm{~S}) \# 1 & 109.82(9) \\ \mathrm{C}(1 \mathrm{~S})-\mathrm{C}(2 \mathrm{~S})-\mathrm{C}(3 \mathrm{~S}) & 109.88(10) \\ & \end{array}$

Symmetry transformations used to generate equivalent atoms:

$\# 1-\mathrm{x},-\mathrm{y}+1,-\mathrm{z}$ 
Table S13. Anisotropic displacement parameters $\left(\AA^{2} \times 10^{3}\right)$ for $\mathbf{6 S b}$. The anisotropic displacement factor exponent takes the form: $-2 \pi^{2}\left[\mathrm{~h}^{2} \mathrm{a}^{* 2} \mathrm{U}^{11}+\ldots+2 \mathrm{~h} \mathrm{k} \mathrm{a}^{*} \mathrm{~b}^{*} \mathrm{U}^{12}\right]$

\begin{tabular}{|c|c|c|c|c|c|c|}
\hline & $\mathrm{U}^{11}$ & $\mathrm{U}^{22}$ & $\mathrm{U}^{33}$ & $\mathrm{U}^{23}$ & $\mathrm{U}^{13}$ & $\mathrm{U}^{12}$ \\
\hline $\mathrm{Sb}$ & $33(1)$ & $39(1)$ & $19(1)$ & $-1(1)$ & $6(1)$ & $10(1)$ \\
\hline $\mathrm{Cl}(1)$ & $37(1)$ & $47(1)$ & $38(1)$ & $10(1)$ & $5(1)$ & $7(1)$ \\
\hline $\mathrm{Cl}(2)$ & $65(1)$ & $33(1)$ & $44(1)$ & $-2(1)$ & $4(1)$ & $-1(1)$ \\
\hline $\mathrm{P}$ & $25(1)$ & $28(1)$ & $15(1)$ & $1(1)$ & $4(1)$ & $3(1)$ \\
\hline $\mathrm{N}(1)$ & $26(2)$ & $40(3)$ & $14(2)$ & $2(2)$ & $4(2)$ & $2(2)$ \\
\hline $\mathrm{N}(2)$ & $28(2)$ & $32(2)$ & $18(2)$ & $1(2)$ & $6(2)$ & $6(2)$ \\
\hline $\mathrm{C}(1)$ & $26(3)$ & $45(3)$ & $18(2)$ & $0(2)$ & $2(2)$ & $7(2)$ \\
\hline $\mathrm{C}(2)$ & $28(2)$ & $25(3)$ & $17(2)$ & $-2(2)$ & $5(2)$ & $1(2)$ \\
\hline $\mathrm{C}(3)$ & $27(2)$ & $29(3)$ & $11(2)$ & $3(2)$ & $4(2)$ & $3(2)$ \\
\hline $\mathrm{C}(4)$ & $27(2)$ & $24(3)$ & $22(2)$ & $0(2)$ & $2(2)$ & $-1(2)$ \\
\hline $\mathrm{C}(5)$ & $32(3)$ & $52(4)$ & $25(3)$ & $11(2)$ & $4(2)$ & $13(3)$ \\
\hline $\mathrm{C}(11)$ & $26(2)$ & $28(3)$ & $25(2)$ & $-1(2)$ & $6(2)$ & $3(2)$ \\
\hline $\mathrm{C}(12)$ & $113(6)$ & $36(4)$ & $35(3)$ & $-2(3)$ & $30(4)$ & $-10(4)$ \\
\hline $\mathrm{C}(13)$ & $147(8)$ & $45(4)$ & $44(4)$ & $2(3)$ & $33(5)$ & $-20(5)$ \\
\hline $\mathrm{C}(14)$ & $87(5)$ & $29(3)$ & $52(4)$ & $2(3)$ & $10(4)$ & $-9(3)$ \\
\hline$C(15)$ & $99(6)$ & $37(4)$ & $60(4)$ & $-18(3)$ & $22(4)$ & $-9(4)$ \\
\hline$C(16)$ & $66(4)$ & $41(4)$ & $39(3)$ & $-11(3)$ & $14(3)$ & $-10(3)$ \\
\hline $\mathrm{C}(21)$ & $28(2)$ & $27(3)$ & $20(2)$ & $-2(2)$ & $2(2)$ & $5(2)$ \\
\hline $\mathrm{C}(22)$ & $50(3)$ & $34(3)$ & $33(3)$ & $6(3)$ & $-2(2)$ & $-9(3)$ \\
\hline $\mathrm{C}(23)$ & $59(4)$ & $37(4)$ & $44(3)$ & $-2(3)$ & $-11(3)$ & $-17(3)$ \\
\hline$C(24)$ & $39(3)$ & $56(4)$ & $41(3)$ & $-22(3)$ & $-10(3)$ & $6(3)$ \\
\hline $\mathrm{C}(25)$ & $42(3)$ & $87(5)$ & $22(3)$ & $-18(3)$ & $3(2)$ & $3(3)$ \\
\hline$C(26)$ & $33(3)$ & $68(4)$ & $22(2)$ & $-4(3)$ & $9(2)$ & $-5(3)$ \\
\hline $\mathrm{C}(31)$ & $25(2)$ & $44(3)$ & $14(2)$ & $8(2)$ & $6(2)$ & $5(2)$ \\
\hline $\mathrm{C}(32)$ & $32(3)$ & $50(4)$ & $22(2)$ & $10(2)$ & $8(2)$ & $5(3)$ \\
\hline $\mathrm{C}(33)$ & $39(3)$ & $67(5)$ & $34(3)$ & $21(3)$ & $9(3)$ & $14(3)$ \\
\hline $\mathrm{C}(34)$ & $32(3)$ & $85(5)$ & $23(3)$ & $15(3)$ & $1(2)$ & $4(3)$ \\
\hline $\mathrm{C}(35)$ & $35(3)$ & $81(5)$ & $21(3)$ & $-5(3)$ & $2(2)$ & $-5(3)$ \\
\hline$C(36)$ & $37(3)$ & $58(4)$ & $21(2)$ & $-1(2)$ & $3(2)$ & $3(3)$ \\
\hline $\mathrm{C}(37)$ & $65(4)$ & $36(4)$ & $39(3)$ & $11(3)$ & $3(3)$ & $3(3)$ \\
\hline $\mathrm{C}(38)$ & $107(7)$ & $61(5)$ & $66(5)$ & $-9(4)$ & $6(5)$ & $24(5)$ \\
\hline
\end{tabular}




$\begin{array}{lcccccc}\mathrm{C}(39) & 86(6) & 65(5) & 68(5) & 8(4) & -1(4) & -28(4) \\ \mathrm{C}(40) & 80(5) & 51(4) & 45(4) & -17(3) & -17(3) & 5(4) \\ \mathrm{C}(41) & 237(14) & 49(5) & 88(7) & 2(5) & 65(8) & 6(7) \\ \mathrm{C}(42) & 117(7) & 78(6) & 69(5) & -36(5) & 8(5) & 17(5) \\ \mathrm{C}(51) & 27(3) & 38(3) & 20(2) & 10(2) & 7(2) & 9(2) \\ \mathrm{C}(52) & 36(3) & 44(3) & 27(3) & 17(2) & 5(2) & 5(3) \\ \mathrm{C}(53) & 28(3) & 63(4) & 38(3) & 18(3) & 12(2) & 9(3) \\ \mathrm{C}(54) & 38(3) & 68(5) & 42(3) & 23(3) & 21(3) & 19(3) \\ \mathrm{C}(55) & 54(4) & 53(4) & 28(3) & 8(3) & 12(3) & 28(3) \\ \mathrm{C}(56) & 39(3) & 42(3) & 22(2) & 8(2) & 7(2) & 13(3) \\ \mathrm{C}(57) & 39(3) & 49(4) & 41(3) & 2(3) & 9(3) & -7(3) \\ \mathrm{C}(58) & 61(4) & 64(5) & 50(4) & 3(3) & -14(3) & -12(4) \\ \mathrm{C}(59) & 48(4) & 45(4) & 78(5) & -5(4) & -14(3) & -4(3) \\ \mathrm{C}(60) & 53(4) & 38(4) & 48(3) & -8(3) & 9(3) & 9(3) \\ \mathrm{C}(61) & 108(7) & 67(5) & 56(4) & 2(4) & 21(4) & -26(5) \\ \mathrm{C}(62) & 122(7) & 58(5) & 50(4) & -10(4) & -1(4) & -11(5)\end{array}$

